\title{
On a conjecture concerning minus parts in the style of Gross
}

\author{
by \\ C. Greither (Neubiberg) and R. KuČera (Brno)
}

Introduction. In the eighties, B. Gross (see [5]) introduced a conjecture which is close to Stark's conjectures inasmuch as it postulates a link between $L$-values and regulators, but differs from Stark's conjectures in a very important aspect: the regulators are not complex numbers, arising as determinants of logarithms of certain algebraic numbers, but they lie in an appropriate quotient of the augmentation filtration of $\mathbb{Z}[G]$, where $G$ is the Galois group of the abelian field extension $K / F$ under consideration, and they are obtained as determinants of matrices made up from certain local Artin symbols.

In a previous version of [1], David Burns formulated a conjecture which combines Stark-type and Gross-type conjectures. (Note: in the most recent version [1], this is formulated not as a conjecture but as a conditional result (Corollary 4.1) assuming the equivariant Tamagawa number conjecture for an appropriate motive. This result requires a very sophisticated proof.) We sketch Burns's conjecture for an abelian extension $K / \mathbb{Q}$ now; this involves two steps. One starts out with a Stark unit $\eta_{K}$ (whose existence is proven in this case, not just a conjecture), and then one obtains a description of the "position of $\eta_{K}$ inside an appropriate exterior power of $\mathcal{O}_{K}^{\times}$" in terms of a Gross regulator. At the first stage, the Stark unit is essentially determined by a classical regulator, that is, a determinant involving the logarithms of the conjugates of $\eta_{K}$. At the second stage, the Gross regulator is an algebraic object living in a subquotient of an integral group ring. (The whole setup generalizes to base fields other than $\mathbb{Q}$.) For details, see $\S 1$.

In subsequent work of Hayward ([6], [7]), where Burns's conjectures are discussed and in some cases proved, another conjecture arises which may be considered as the "minus part" of Burns's conjecture for extensions $K / F$ where $F$ is an imaginary quadratic field and $K$ is absolutely abelian. (The

2000 Mathematics Subject Classification: Primary 11R20.

Key words and phrases: Stark units, regulators, Gross conjecture on tori. 
methods in Hayward's proofs are much more accessible than those of [1].) We will explain this in $\S 1$ without striving for maximum generality, so as to keep things a little simpler. This "Minus Conjecture" equates, up to explicit constant factors, the leading term of a Stickelberger element and a regulator constructed from $S$-units in the minus part. We hasten to mention two things: firstly, our Minus Conjecture is in fact a special case of what is called "the conjecture of Gross on tori", on which not much seems to be known, and secondly we are indebted to Henri Darmon for his suggestion that we might look at leading terms of Stickelberger elements in the minus part. Let us also remark that Darmon deals with an interesting analogous situation in [3] where $F$ is a real quadratic field.

The Minus Conjecture (MC) is intimately linked to the conjecture of Burns (B) for $K^{+} / \mathbb{Q}$ and $K / F$ respectively: if we assume the validity of (B) for $K^{+} / \mathbb{Q}$, then the Minus Conjecture can be shown to imply the validity of (B) for $K / F$ (this is a result of Hayward, aptly called "base change for Conjecture (B)"), and the converse implication, which entails a kind of division argument, works under some hypotheses (Theorem 2.5). We will review Hayward's argument in detail in $\S 2$, in order to make clear just when the division argument is possible. The idea is, very crudely speaking, that (B) for $K^{+} / \mathbb{Q}$ is the "plus part of (B) for $K / F$ ", and the Minus Conjecture (MC) is "the minus part of (B) for $K / F$ ", and the problem is to neatly separate the plus and minus parts.

We repeat that the division argument does not always work. The problem is simply "division of zero by zero". Our main objective in the second part of this paper $(\S \S 5-8)$ is thus to find a direct proof of the Minus Conjecture, which appears to be deeper than (B). Hayward proved the above-mentioned instances of (B) by a nice argument involving Euler systems and a matrix-tree theorem. As things stand, this is not always sufficient for $(\mathrm{MC})$, although it does lead the way to our proof of the weaker conjecture (VOC) on the order of vanishing (see below and §4). In our direct proof of (MC), we are only able to handle $s=1$ and $s=2$, but still have to use (along with a lot of calculation) the Gross-Koblitz formula in $\S 7$. The situation in [3] is somewhat similar: the order of vanishing (Theorem 4.2, loc.cit.) is easier to obtain than the results on the leading coefficient.

Actually, we impose some more hypotheses in order to simplify things: We assume $K / F$ elementary $l$-abelian with $l$ a fixed odd prime; we suppose that $K=K^{+} F$, and $K^{+}$is ramified at the primes $p_{1}, \ldots, p_{s}$ that are distinct from $l$, and we also suppose that all $p_{i}$ split in $F$. Let $w_{F}, f$ and $h_{F}$ denote the number of roots of unity, the conductor and the class number of $F$, respectively. We make the blanket assumption that $l \nmid w_{F}$. Then we can prove (MC) for $s=1$ and $l \nmid f$ (see $\S 3$ ), and for cyclic $K, s=2$ and $l \nmid f h_{F}$ 
(Theorem 8.9). We also have a positive result for $s=2$ and noncyclic $K$ (Theorem 8.8), but this requires that one of $p_{1}, p_{2}$ is an $l$ th power modulo the other prime, and again $l \nmid f h_{F}$.

The Vanishing Order Conjecture (VOC), which is a weakened form of (MC), says that the Stickelberger element associated to $K$ is contained in the power $I_{G}^{s}$, where $I_{G}$ is the augmentation ideal of $\mathbb{Z}_{l}[G]$ and $s$ is the number of ramified primes in $K^{+} / \mathbb{Q}$. We prove this under some assumptions in $\S 4$ for all $s$. This shows that the invariant predicted to vanish by (MC) lives in the filtration quotient $I_{G}^{s} / I_{G}^{s+1}$.

It should be said that the case $s=1$ of (MC) can already be deduced from our Theorem 2.5 together with [7]. The latter is an unpublished Ph.D. thesis, and we decided to present our own approach anyway, since we also feel that it might be of independent arithmetic interest. Hayward's argument makes essential use of elliptic units. While this is quite natural in the setting of [7], one may argue that in our case even the top field $K$ is abelian over $\mathbb{Q}$, so one should try to stick to the cyclotomic framework all the way, and we show that this is possible.

In a forthcoming paper [4] we will explain how to prove (MC) for an arbitrary $s$ under the assumption that $l \geq 3(s+1)$ and $l \nmid w_{F} h_{F}$.

Notation will be introduced in $\S 1$ and further along as needed. We only mention here that $X / l$ means $X / l X$ or $X / X^{l}$ depending on whether the abelian group $X$ is written additively or multiplicatively.

Acknowledgements. The second author was supported within the project MSM0021622409 of the Ministry of Education of the Czech Republic. The first author acknowledges support from the DFG.

1. The setup, and statement of the conjectures. We fix an odd prime $l$ for the entire paper. First we state Burns's conjecture (B) on abelian Galois extensions $K / k$ in a situation which is appropriate for our setting, always assuming that $l \nmid w_{k}$. We only look at the case where the parameter $r$ (see [6]) has value 1 . This suggests taking $k=\mathbb{Q}$ and $K$ real, or taking $k$ to be an imaginary quadratic field, since these are the obvious examples of abelian extensions where exactly one infinite place is totally split. To keep things simple we also assume $G=\operatorname{Gal}(K / k)$ is $l$-elementary. Let $S$ be a nonempty finite set of finite places of $k$ including all places that ramify in $K$, and let $s$ denote the cardinality of $S$. Stark's conjecture in its strong form for rank one is known to be true for $k$ the rationals or imaginary quadratic, and we will write $\eta_{K / k, S}$ for the Stark unit. For more details see [6] or [11]. Note that $\eta_{K / k, S}$ depends on the choice of the set $S$, and also in a harmless way on the choice of a place at infinity for $K$. Note moreover that in general $\eta_{K / k, S}$ only belongs to $U_{S}(K)^{1 / w_{K}}$, so certainly belongs to $\mathbb{Z}_{l} \otimes_{\mathbb{Z}} U_{S}(K)$. We always 
suppose $K$ given as a subfield of $\mathbb{C}$, and then there is the obvious choice of the infinite place.

In the statement of the conjecture we follow [6], except that we consistently eliminate the auxiliary set $T$ which is used there to make certain unit groups torsion-free. The price for this is a denominator $w_{k}$, and we pay it gladly since all our conjectures amount to equalities that take place in groups of exponent $l$, and the absence of $T$ does help.

There is a linear map

$$
\begin{gathered}
\operatorname{Reg}_{K / k, S}: \bigwedge_{\mathbb{Z}}^{s-1} U_{S}(k) \rightarrow I_{G}^{s-1} / I_{G}^{s}, \\
u_{1} \wedge \cdots \wedge u_{s-1} \mapsto \operatorname{det}\left(r_{v}\left(u_{i}\right)-1\right)_{1 \leq i \leq s-1, v \in S^{*}} .
\end{gathered}
$$

Here $r_{v}$ is the local reciprocity map $x \mapsto\left(x, K_{w} / k_{v}\right) \in G_{v} \subseteq G$ (note that the choice of the place $w$ in $K$ above $v$ does not matter), and $S^{*}$ denotes $S$ with any one place deleted. This regulator map is well-defined up to sign; we will specify the sign shortly, before stating the central conjecture.

We note at once that $r_{v}$ is trivial on roots of unity in $k$, since $w_{k}$ is assumed to be coprime to $|G|$.

To obtain a regulator from the regulator map, one has (in contrast with Stark's conjecture) to introduce an extra parameter running over a Hom group. For any $G$-module $X$ and any $\varphi \in \operatorname{Hom}_{\mathbb{Z}[G]}(X, \mathbb{Z}[G])$, let $\varphi^{1} \in$ $\operatorname{Hom}_{\mathbb{Z}}\left(X^{G}, \mathbb{Z}\right)$ be defined by the property that $\varphi(u)=\varphi^{1}(u) \cdot \sum_{\sigma \in G} \sigma$ for all $u \in X^{G}$. Then $\varphi^{1}$ canonically induces a linear map $\bigwedge_{\mathbb{Z}}^{s} X^{G} \rightarrow \bigwedge_{\mathbb{Z}}^{s-1} X^{G}$ which will again be written $\varphi^{1}$, to wit

$$
\varphi^{1}\left(x_{1} \wedge \cdots \wedge x_{s}\right)=\sum_{i=1}^{s}(-1)^{i+1} \varphi^{1}\left(x_{i}\right) \cdot\left(x_{1} \wedge \cdots \wedge x_{i-1} \wedge x_{i+1} \wedge \cdots \wedge x_{s}\right) .
$$

We now pick any $\mathbb{Z}$-basis $u_{1}, \ldots, u_{s}$ of $U_{S}(k) / U_{S}(k)_{\text {tor }}$ and we define

$$
\operatorname{Reg}_{K / k, S}^{\varphi}=\operatorname{Reg}_{K / k, S}\left(\varphi^{1}\left(u_{1} \wedge \cdots \wedge u_{s}\right)\right) \in I_{G}^{s-1} / I_{G}^{s}
$$

for all $\varphi \in \operatorname{Hom}_{\mathbb{Z}[G]}\left(U_{S}(K), \mathbb{Z}[G]\right)$. Note that this is well-defined (independent of the choice of the basis) up to sign.

We have to fix the sign of the regulator before we state Burns's conjecture. For this we use ad hoc terminology. Let us fix an ordering $v_{1}, \ldots, v_{s}$ of the set $S$. (In contrast to Hayward's work, the infinite place is not counted as a member of $S$.) An independent system $u_{1}, \ldots, u_{s}$ of $S$-units is called adapted to the given ordering of $S$ (or just adapted, in context) if $u_{i}$ is a unit outside $v_{i}$ and has positive value at $v_{i}$. A basis $u_{1}, \ldots, u_{s}$ of $U_{S}(k) / U_{S}(k)_{\text {tor }}$ is called well-oriented if the transition matrix from this basis to any adapted system has positive determinant. (In many cases, e.g. if $h_{F}=1$, we will even be able to pick a basis which is itself adapted.) Unless otherwise stated, we always make two assumptions: 
(1) $S$ and the basis $u_{1}, \ldots, u_{s}$ are ordered so that the basis is welloriented.

(2) In the calculation of the regulator, the last place in $S$ is omitted.

We may now state:

Conjecture (B). For all $\varphi \in \operatorname{Hom}_{\mathbb{Z}[G]}\left(U_{S}(K), \mathbb{Z}[G]\right)$ one has

$$
\varphi\left(\eta_{K / k, S}\right) \equiv(-1)^{s+1} \frac{h_{k, S}}{w_{k}} \operatorname{Reg}_{K / k, S}^{\varphi}\left(\bmod I_{G}^{s}\right) .
$$

The letter $\varphi$ here stands for the extension of $\varphi$ in $\operatorname{Hom}_{\mathbb{Z}_{l}[G]}\left(\mathbb{Z}_{l} \otimes U_{S}(K), \mathbb{Z}_{l}[G]\right)$.

The prediction about the sign comes from [1] and is also explained in [7]. Our way of stating things is different from [7] (and a bit more explicit), so we have to show compatibility. [7] uses the $\operatorname{sign} \xi=(-1)^{|T|+1} \operatorname{sign}(R)$ where $R$ is a certain real-valued regulator. Since we work with empty $T$, we just have to show that the sign of $R$ is exactly $(-1)^{s}$ under our assumptions.

The real-valued regulator $R$ used by Hayward is constructed as follows: one takes an ordered basis $u_{1}, \ldots, u_{s}$ as above but a slightly different set of places $v_{0}:=\infty, v_{1}, \ldots, v_{s-1}$ (unfortunately Hayward's indexing is different, starting at 1 ), and one considers the determinant

$$
R=\operatorname{det}\left(-\log \left|u_{i}\right|_{v_{j-1}}\right)_{1 \leq i, j \leq s} .
$$

If one extends $R$ by an $(s+1)$ th column by letting $j$ run up to $j=s+1$, then the resulting matrix has zero row sums. By the usual argument we have

$$
R=(-1)^{s} \operatorname{det}\left(-\log \left|u_{i}\right|_{v_{j}}\right)_{1 \leq i, j \leq s} .
$$

Now if $u_{1}, \ldots, u_{s}$ is an adapted system, the matrix inside the last det is diagonal, with positive entries $a_{i} \log \mathrm{N}\left(v_{i}\right)$ (where $a_{i}$ denotes the valuation of $u_{i}$ at $v_{i}$ ). Thus the determinant is positive, and the same holds if $u_{1}, \ldots, u_{s}$ is well-oriented. This shows $\operatorname{sign}(R)=(-1)^{s}$ and $\xi=(-1)^{s+1}$ as claimed.

In order to show that our version conforms with Hayward's formulation on p. 104 of [6], we also have to remark the following: Hayward's formula is invariant under enlarging $T$, as long as $U_{S, T}(k)$ is assumed torsion-free to begin with. We put in an extra denominator $w_{k, T}$ on the right in his formula, and we claim that then the formula is invariant under changing $T$, without any condition. Indeed, if we replace $T$ by $T^{\prime}=T \cup\{v\}$ ( $v$ some finite place of $k$ outside $S \cup T)$, then $\eta_{K / k, S, T^{\prime}}=\eta_{K / k, S, T}^{\alpha}$ with $\alpha=1-\mathrm{N}(v) \cdot \operatorname{Frob}_{v}^{-1}$, so $\varphi\left(\eta_{K / k, S, T^{\prime}}\right)=\alpha \varphi\left(\eta_{K / k, S, T}\right)$, and we only need to know $\alpha$ modulo $I_{G}$, so we just get the factor $1-\mathrm{N}(v)$. A standard argument using formula (3) in [6] then shows that $h_{k, S, T} \operatorname{Reg}_{K / k, S, T}^{\varphi} / w_{k, T}$ is multiplied by the factor $\mathrm{N}(v)-1$ when $T$ is replaced by $T^{\prime}$. Setting $T=\emptyset$ gives our version, since of course $h_{k, S, \emptyset}=h_{k, S}$ and $w_{k, \emptyset}=w_{k}$.

This shows at once that our version implies Hayward's. But the converse is equally true. For this we point out that one can always take $T=\{v\}$ 
in Hayward's situation so that $l$ does not divide $\mathrm{N}(v)-1$ (here we have to use the assumption that $w_{k}$ is coprime to $l$ ). This ensures that the above argument works both ways. Note in this context that the term $(-1)^{|T|}$ in Hayward's sign definition is due to the fact that the sign changes whenever an element is put into or taken out of $T$, as can be seen by the preceding argument.

We will be concerned with Conjecture (B) in two concrete cases which we now describe. Let $F$ be an imaginary quadratic field of conductor $f$. Let $h_{F}$ denote the class number of $F$ and $w_{F}$ the number of roots of unity in $F$. We recall that we assume $l \nmid w_{F}$, and we stress that this hypothesis will be in force throughout the paper. We always assume that $K$ is absolutely abelian, contains $F$, and $K^{+}$is elementary $l$-abelian over $\mathbb{Q}$. Then $K=$ $F K^{+}$and $G=\operatorname{Gal}(K / F)$ may be identified with $\operatorname{Gal}\left(K^{+} / \mathbb{Q}\right)$. Let $\tau$ always stand for complex conjugation. Then both $\operatorname{Gal}\left(K / K^{+}\right)$and $\operatorname{Gal}(F / \mathbb{Q})$ can be identified with $\{1, \tau\}$. Let $m$ be the conductor of $K^{+}$. We also assume that there is no wild ramification in $K^{+} / \mathbb{Q}$. Then $m$ has the form $m=p_{1} \cdots p_{s}$ where the $p_{i}$ are all congruent to $1 \bmod l$. Finally, we assume all $p_{i}$ are split in $F$.

TheOREM 1.1. Under all these assumptions, Conjecture (B) is true for $K^{+} / \mathbb{Q}$ with $S=\left\{p_{1}, \ldots, p_{s}\right\}$ (the minimal possible choice of $S$ ).

Proof. This is due to Hayward. In the published work [6], this result can be found up to sign (see Theorem 5.10 and Remark 5.11 there). The sharp version including the sign is only proved in Hayward's thesis (top of page 98). We have to explain the role of the auxiliary integer $b$ by which both sides of the conjecture are multiplied in [6]. It has to satisfy two conditions: $b$ must be a multiple of $w_{k}$, and there must be a section $s$ of the natural epimorphism $R \rightarrow \mathrm{Cl}_{k}$ such that $s^{b}$ is a homomorphism, where $R$ denotes the ray class group of conductor $p_{1} \cdots p_{s}$ in $k$. Since $k=\mathbb{Q}$ here, the class group is trivial, and the second condition is void; so we may take $b=w_{\mathbb{Q}}=2$, which is prime to $l$, and we may cancel $b$ in Hayward's theorem on both sides.

We make a few comments:

(i) As a basis for $U_{S}(\mathbb{Q})$ modulo torsion one can take the set $\left\{p_{1}, \ldots, p_{s}\right\}$. Note this is well-oriented (with the obvious ordering of $S$ ) and even adapted.

(ii) The Stark unit appears as $\mathrm{N}_{\mathbb{Q}\left(\zeta_{m}\right) / K^{+}}\left(1-\zeta_{m}\right)^{1 / 2}$ where $\zeta_{m} \mapsto e^{2 \pi i / m}$ fixes the choice of place at infinity.

(iii) The factor preceding the regulator on the right is simply $h_{\mathbb{Q}, S} / w_{\mathbb{Q}}$ $=1 / 2$.

On the upper level we have: 
Theorem 1.2. If $l \nmid h_{F}$ then Conjecture (B) is true for $K / F$ with $S=S_{F}$ the set of places above any of the $p_{i}, i=1, \ldots, s$ (the minimal possible choice of $S$ ).

Proof. This is again due to Hayward. He actually proves much more, using elliptic units; the big field $K$ only needs to be abelian over $k$, not necessarily over $\mathbb{Q}$. The requirements on the integer $b$ have already been explained, but here $k=F$, and we have to be more careful. Let $b$ be $w_{F}$ multiplied with the greatest factor of $\prod_{i=1}^{s}\left(p_{i}-1\right)$ that is prime to $l$. Then $b$ annihilates the non-l-part of the kernel of $R \rightarrow \mathrm{Cl}_{F}$, and $b$ is prime to $l$. Since we assumed $h_{F}$ to be prime to $l$, there is a section $s: \mathrm{Cl}_{F} \rightarrow R$ with image contained in the non-l-part of $R$. Then $s^{b}$ is a homomorphism, so Hayward's theorem (p. 98 in [7]) applies again, and we may cancel $b$ as in the proof of Theorem 1.1.

Again, a few comments:

(iv) Now the set $S_{F}$ has cardinality $2 s$, so we are looking at an equation in $I_{G}^{2 s-1} / I_{G}^{2 s}$.

(v) In the particular case that interests us in $\S 3$ and $\S \S 5-8$, we will fix an explicit basis of $U_{S}(F)$.

(vi) One can express $\eta_{K / F, S}$ in terms of $\eta_{K^{+} / \mathbb{Q}, S}$; this will be made precise at an appropriate later moment.

(vii) The denominator is legal since we are assuming $l \nmid w_{F}$.

We now turn to the Minus Conjecture (MC). To state it, we have to introduce certain Stickelberger elements.

The notation $\sum_{a \bmod ^{\times}{ }_{n}}$ means that the sum runs over all $a=1, \ldots, n-1$ which are coprime to $n$. Let $\sigma_{a}$ denote the automorphism $\zeta_{f m} \mapsto \zeta_{f m}^{a}$ for $a$ coprime to $\mathrm{fm}$. We likewise denote by $\sigma_{a}$ the restriction of this to $K=$ $F K^{+} \subseteq \mathbb{Q}\left(\zeta_{f m}\right)$. We define

$$
\Theta_{K}=\frac{1}{f m} \sum_{a \bmod _{f m}}\left(a-\frac{f m}{2}\right) \sigma_{a}^{-1} \in \mathbb{Q}[\operatorname{Gal}(K / \mathbb{Q})] .
$$

We note that $\tau \in \mathbb{Q}[\operatorname{Gal}(K / \mathbb{Q})]$ is just $\sigma_{-1}$, and comparing coefficients shows that $\Theta_{K}$ is a multiple of $1-\tau$, more precisely,

$$
\Theta_{K}=(1-\tau) \widetilde{\Theta}_{K}, \quad \widetilde{\Theta}_{K}=\frac{1}{f m} \sum_{\substack{a \bmod ^{\times} f m \\ \sigma_{a} \mid F=1}}\left(a-\frac{f m}{2}\right) \sigma_{a}^{-1} .
$$

Note that $\widetilde{\Theta}_{K}$ is now in $\mathbb{Q}[\operatorname{Gal}(K / F)]$.

We also need a regulator built from minus-units. For each $i \in\{1, \ldots, s\}$ pick a prime ideal $\mathfrak{p}_{i}$ above $p_{i}$ in $F$. Let $S^{\prime}$ be the ordered set $\left(\mathfrak{p}_{1}, \ldots, \mathfrak{p}_{s}\right)$. The abelian group $\left(U_{S}(F) / U_{S}(F)_{\text {tor }}\right)^{1-\tau}$ is free of rank $s$. We choose an ordered basis for it, $\left(u_{1}^{1-\tau}, \ldots, u_{s}^{1-\tau}\right)$ say. We again have a notion of well-orientedness 
as follows. For each $i$ we choose a positive power of $\mathfrak{p}_{i}$ which is principal, and a generator $x_{i}$ of it. Then the family $x_{1}^{1-\tau}, \ldots, x_{s}^{1-\tau}$ is a $\mathbb{Q}$-basis of $\mathbb{Q} \otimes\left(U_{S}(F) / U_{S}(F)_{\text {tor }}\right)^{1-\tau}$ (an analog of the "adapted systems" we used before), and we decree that $\left(u_{1}^{1-\tau}, \ldots, u_{s}^{1-\tau}\right)$ is well-oriented with respect to the ordered set $S^{\prime}$ if $\left(x_{1}^{1-\tau}, \ldots, x_{s}^{1-\tau}\right)$ can be obtained by a $\mathbb{Q}$-linear transformation with positive determinant from the ordered set $\left(u_{1}^{1-\tau}, \ldots, u_{s}^{1-\tau}\right)$. All this simplifies a lot when $h_{F}=1$. Then one takes $u_{i}$ to be a generator of $\mathfrak{p}_{i}$.

No parameter $\varphi$ is needed, and no place has to be omitted in the following definition:

$$
\operatorname{Reg}_{K, S}^{-}=\operatorname{det}\left(r_{w}\left(u_{i}^{1-\tau}\right)-1\right)_{1 \leq i \leq s, w \in S^{\prime}} \in I_{G}^{s} / I_{G}^{s+1} .
$$

This is indeed well-defined. In order to state the Minus Conjecture, we repeat our assumptions for convenience: $K=K^{+} F, l \nmid w_{F}, K^{+} / \mathbb{Q}$ is l-elementary with Galois group $G$, exactly $s$ rational primes ramify (tamely) in $K^{+}$, and all these primes are split in $F$.

Conjecture (MC). Let $\left(u_{i}^{1-\tau}\right)_{i}$ be an ordered basis of the minus units as above which is well-oriented with respect to $S^{\prime}$. Then

$$
\widetilde{\Theta}_{K} \equiv-\frac{h_{F, S}}{w_{F}} \operatorname{Reg}_{K, S}^{-}\left(\bmod I_{G}^{s+1}\right) .
$$

We will call this the "Minus Conjecture" in the following.

We point out that in the notation of [6] (p. 118), $\widetilde{\Theta}_{K}=-\frac{1}{2} \Theta(0, \omega)$ (note the minus sign, which comes from the usual formula linking an Lvalue at 0 to a generalized Bernoulli number), so (MC) reads as $\Theta(0, \omega) \equiv$ $\left(2 h_{F, S} / w_{F}\right) \operatorname{Reg}_{K, S}^{-}$, and the constant $2 h_{F, S} / w_{F}$ should be interpreted as $\left(h_{F, S} / w_{F}\right) /\left(h_{\mathbb{Q}} / w_{\mathbb{Q}}\right)$, which agrees with the general idea that $(\mathrm{MC})$ is "the quotient of $(\mathrm{B})$ for $K / F$ by (B) for $K^{+} / \mathbb{Q}$." There is one intentional discrepancy with [6]:

REMARK. In our minus regulator we take a basis of $\left(U_{S}(F) / U_{S}(F)_{\text {tor }}\right)^{1-\tau}$ whereas Hayward takes a basis of the group $\left(U_{S}(F) / U_{S}(F)_{\text {tor }}\right)^{-}$, which may be slightly larger. This makes our regulator larger by the index of the smaller group inside the latter; and so we get rid of the index factor which is needed in the statements of Proposition 7.2 and Conjecture 7.4 in [6].

The following important consequence of the Minus Conjecture is much easier to state, and also easier to prove (see Theorem 4.4).

Conjecture (VOC). The element $\widetilde{\Theta}_{K}$ always lies in the $s$ th power of the augmentation ideal $I_{G}$.

2. Base change for (B) and the division argument for (MC). In simple terms, we are going to show: If (MC) holds, then Theorem 1.2 is a direct consequence of Theorem 1.1 (it would be slightly misleading to say 
that the one implies the other, since both statements are true); and under specific conditions this argument can be reversed, leading to a proof of (MC). The former part of this is due to Hayward. We explain the argument in detail for the reader's convenience before discussing the converse implication.

We keep all assumptions and notations from the previous section.

Proposition 2.1 (Hayward). We have a base change property for Conjecture (B), that is: Theorem 1.2 can be deduced from Theorem 1.1 and the validity of (MC).

Proof. The main idea is that both sides in the statement of (B) for $K / F$ can be conveniently written as the product of two factors.

In [6] Hayward defines a twisted Stickelberger element $\Theta_{K / K^{+} / \mathbb{Q}, S}(0, \omega)$. One can check that it equals $-2 \widetilde{\Theta}_{K}$. By the formula (13) of [6] it satisfies the product formula

$$
\Theta_{K / F, S}^{\prime}(0)=\Theta_{K / K^{+} / \mathbb{Q}, S}(0, \omega) \cdot \Theta_{K^{+} / \mathbb{Q}, S}^{\prime}(0),
$$

where the equivariant $L$-functions $\Theta_{K / F, S}(z)$ and $\Theta_{K^{+} / \mathbb{Q}, S}(z)$ are defined for instance in [11, Chapter IV, §1]. Note that the idempotent $e_{\omega \chi}$ two lines below formula (13) of [6] should read $e_{\chi}$.

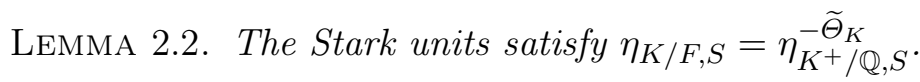

Proof. This was proved by Hayward under Hypothesis 7.1 of [6]. An argument is necessary here as we are not assuming that hypothesis.

Let $\infty_{1}$ be an infinite place of $K^{+}$; we identify it with the place of $K$ above it. Let $v$ be a place of $K^{+}$above $p_{1}$, and $w$ be a place of $K$ above $v$. We denote by $\bar{w}$ the $K / K^{+}$conjugate of $w$ which may or may not be equal to $w$.

Similarly to $[6]$ we deduce the following two formulas:

$$
\begin{aligned}
\lambda_{K}\left(\eta_{K^{+} / \mathbb{Q}}^{-\widetilde{\Theta}_{K}}\right) & =-\Theta_{K^{+} / \mathbb{Q}, S}^{\prime}(0) \widetilde{\Theta}_{K} \cdot\left(2 \infty_{1}-w-\bar{w}\right) \\
& =\Theta_{K / F, S}^{\prime}(0) \cdot\left(\infty_{1}-w / 2-\bar{w} / 2\right), \\
\lambda_{K}\left(\eta_{K / F}\right) & =\Theta_{K / F, S}^{\prime}(0) \cdot\left(\infty_{1}-w\right) .
\end{aligned}
$$

We want to conclude that the arguments of $\lambda_{K}$ in two preceding formulas are equal but we cannot do so directly since $\lambda_{K}$ is not injective in general.

Let $e_{K} \in \mathbb{C}[G]$ be the idempotent which is the sum of all $e_{\chi}$ such that $\chi$ is nontrivial on all decomposition groups of primes in $S$. We claim that

$$
e_{K}\left(\infty_{1}-w / 2-\bar{w} / 2\right)=e_{K}\left(\infty_{1}-w\right) .
$$

If $w=\bar{w}$, this is trivial. If not, suppose $e_{\chi}$ is one of the idempotents whose sum is $e_{K}$. Then $\chi$ must be nontrivial on the decomposition group of $w$ and of $\bar{w}$. But then $e_{K} w=e_{K} \bar{w}=0$.

Since $\lambda_{K}$ is injective in the $e_{K}$-part, we have proved the $e_{K}$-part of the lemma. We will be done if we establish that both sides of the equality are 
already in the $e_{K}$-part. The support of an element $x$ of a $\mathbb{C}[G]$-module is by definition the set of all $\chi \in \widehat{G}$ with $e_{\chi} x \neq 0$. We know the following: $\eta_{K / F}$ has the same support as $\Theta_{K / F, S}^{\prime}(0)$ and $\eta_{K^{+} / \mathbb{Q}}$ has the same support as $\Theta_{K^{+} / \mathbb{Q}, S}^{\prime}(0)$. Then using the product formula stated before Lemma 2.2 we deduce that both sides of the equality in Lemma 2.2 have the same support, in particular they are both in the $e_{K}$-part.

From the preceding lemma it follows that $\varphi\left(\eta_{K / F, S}\right)=-\widetilde{\Theta}_{K} \varphi\left(\eta_{K^{+} / \mathbb{Q}, S}\right)$ for all $\varphi \in \operatorname{Hom}_{\mathbb{Z}[G]}\left(U_{S}(K), \mathbb{Z}[G]\right)$.

We fix an ordering of the primes $p_{1}, \ldots, p_{s}$; for each $i$ we fix a prime $\mathfrak{p}_{i}$ above $p_{i}$ in $F$ and we choose the ordering $\left(\mathfrak{p}_{1}, \ldots, \mathfrak{p}_{s}, \mathfrak{p}_{1}^{\tau}, \ldots, \mathfrak{p}_{s}^{\tau}\right)$ on $S$.

Lemma 2.3. Recall $S^{\prime}=\left\{\mathfrak{p}_{1}, \ldots, \mathfrak{p}_{s}\right\}$. If $\mathbf{u}^{\prime}=\left(u_{1}, \ldots, u_{s}\right)$ is an ordered basis of $U_{S^{\prime}}(F) / U_{S^{\prime}}(F)_{\text {tor }}$, then $\mathbf{u}=\left(u_{1}, \ldots, u_{s}, p_{1}, \ldots, p_{n}\right)$ is an ordered basis of $U_{S}(F) / U_{S}(F)_{\text {tor }}$, and $\mathbf{u}$ is well-oriented with respect to $S$ iff $\mathbf{u}^{\prime}$ is well-oriented with respect to $S^{\prime}$.

Proof. We begin by noting that $U_{S}(F) / U_{S}(F)$ tor is the direct sum of $U_{S^{\prime}}(F) / U_{S^{\prime}}(F)_{\text {tor }}$ and $U_{S}(\mathbb{Q}) / U_{S}(\mathbb{Q})_{\text {tor }}=\left\langle p_{1}, \ldots, p_{s}\right\rangle$. Pick $x_{i} \in F$ so that $x_{i}$ generates a positive power of $\mathfrak{p}_{i}$. Then $\mathbf{x}=\left(x_{1}, \ldots, x_{s}, x_{1}^{\tau}, \ldots, x_{s}^{\tau}\right)$ is an adapted system with respect to $S$, and one checks that the transition from it to $\left(x_{1}, \ldots, x_{s}, p_{1}, \ldots, p_{s}\right)$ has positive determinant. So the transition from $\mathbf{u}$ to $\mathbf{x}$ has positive determinant iff the transition from $\mathbf{u}^{\prime}$ to $\left(x_{1}, \ldots, x_{n}\right)$ does.

We continue the proof of Proposition 2.1, assuming that $S, S^{\prime}, u_{1}, \ldots, u_{s}$ have been chosen so that $\mathbf{u}$ is well-oriented. We write $u_{s+i}$ for $p_{i}(i=$ $1, \ldots, s$ ). We will prove below the following formula (in which the first and third equalities hold by definition):

$$
\begin{aligned}
\operatorname{Reg}_{K / F, S}^{\varphi} & =\operatorname{Reg}_{K / F, S}\left(\varphi^{1}\left(u_{1} \wedge \cdots \wedge u_{2 s}\right)\right) \\
& =\frac{1}{2}(-1)^{s} \operatorname{Reg}_{K, S}^{-} \cdot \operatorname{Reg}_{K^{+} / \mathbb{Q}, S}\left(\varphi^{1}\left(u_{s+1} \wedge \cdots \wedge u_{2 s}\right)\right) \\
& =\frac{1}{2}(-1)^{s} \operatorname{Reg}_{K, S}^{-} \operatorname{Reg}_{K^{+} / \mathbb{Q}, S}^{\varphi} .
\end{aligned}
$$

Let us assume this for a moment. For the ensuing calculation, which takes place in the group $I_{G}^{2 s-1} / I_{G}^{2 s}$, we note that there is a canonical map $I_{G}^{s} / I_{G}^{s+1} \times$ $I_{G}^{s-1} / I_{G}^{s} \rightarrow I_{G}^{2 s-1} / I_{G}^{2 s}$ induced by multiplication. We have

$$
\begin{aligned}
\varphi\left(\eta_{K / F, S}\right) & =-\widetilde{\Theta}_{K} \cdot \varphi\left(\eta_{K^{+} / \mathbb{Q}, S}\right) \\
& =\left(+\frac{h_{F, S}}{w_{F}} \operatorname{Reg}_{K, S}^{-}\right)\left((-1)^{s+1} \frac{1}{2} \operatorname{Reg}_{K^{+} / \mathbb{Q}, S}^{\varphi}\right) \\
& \quad\left(\text { use }(\mathrm{B}) \text { for } K^{+} / \mathbb{Q}, \text { and }(\mathrm{MC})\right) \\
& =(-1)^{s+1} \frac{h_{F, S}}{2 w_{F}} \operatorname{Reg}_{K, S}^{-} \operatorname{Reg}_{K^{+} / \mathbb{Q}, S}^{\varphi} .
\end{aligned}
$$

This is (B) for $K / F$. 
To complete the proof, we need another proposition as announced. We follow Hayward's reasoning.

Proposition 2.4. With all the previously introduced hypotheses,

(*) $\operatorname{Reg}_{K / F, S}\left(\varphi^{1}\left(u_{1} \wedge \cdots \wedge u_{2 s}\right)\right)$

$$
=\frac{1}{2}(-1)^{s} \operatorname{Reg}_{K, S}^{-} \cdot \operatorname{Reg}_{K^{+} / \mathbb{Q}, S}\left(\varphi^{1}\left(u_{s+1} \wedge \cdots \wedge u_{2 s}\right)\right) .
$$

Proof. We have a matrix with row sums in $I_{G}^{2}$, whose rows are indexed by $i=1, \ldots, 2 s$ and the columns are indexed as indicated:

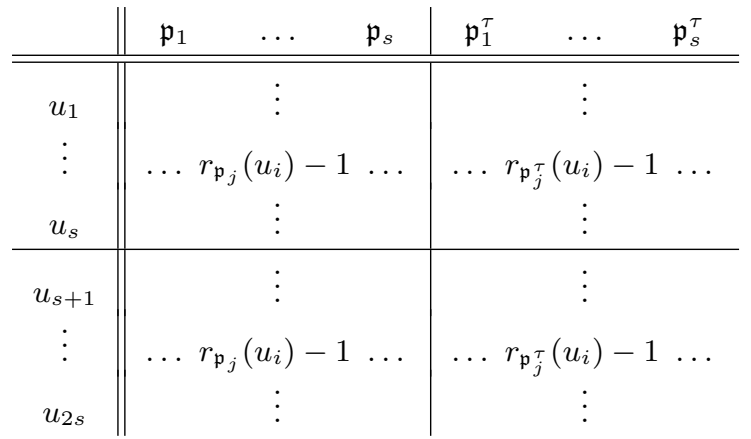

We may use that $r_{\mathfrak{p}_{j}^{\tau}}\left(u_{i}\right)-1=r_{\mathfrak{p}_{j}}\left(u_{i}^{\tau}\right)-1$ and $u_{i}^{\tau}=u_{i}$ if $i>s$. For any $i$, $s<i \leq 2 s$, we have $2 \sum_{j=1}^{s}\left(r_{\mathfrak{p}_{j}}\left(u_{i}\right)-1\right) \in I_{G}^{2}$. But $I_{G} / I_{G}^{2} \simeq G$ has no element of order 2 and so $\sum_{j=1}^{s}\left(r_{\mathfrak{p}_{j}}\left(u_{i}\right)-1\right) \in I_{G}^{2}$. We remove the last column from the matrix and call the resulting matrix $A$ :

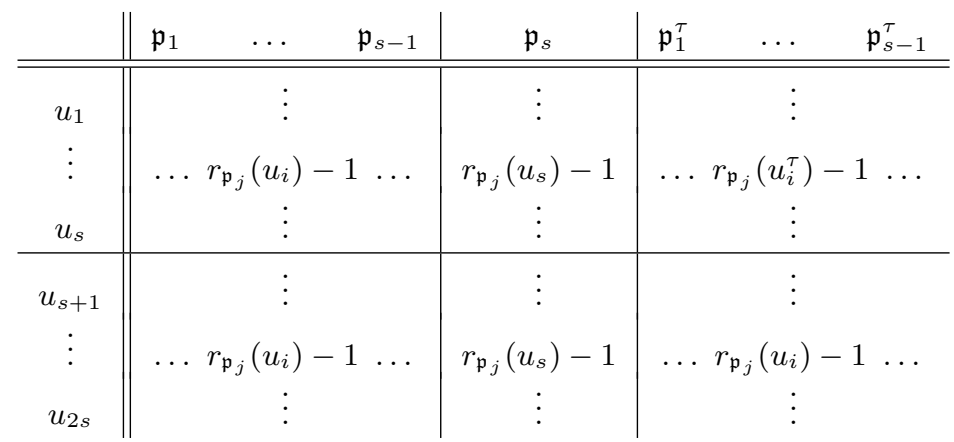

The left hand side of $(*)$ is then $\sum_{i=1}^{2 s}(-1)^{i+1} \varphi\left(u_{i}\right) \operatorname{det}\left(A_{i}\right)$, where $A_{i}$ means $A$ without the $i$ th row. Before we can compute further, we perform some column operations on $A$ : We add all the first $s-1$ columns to the $s$ th one and then for each $i=1, \ldots, s-1$ we subtract the $(i+s)$ th column from the $i$ th one. All computations are done modulo $I_{G}^{2}$, and the outcome is 


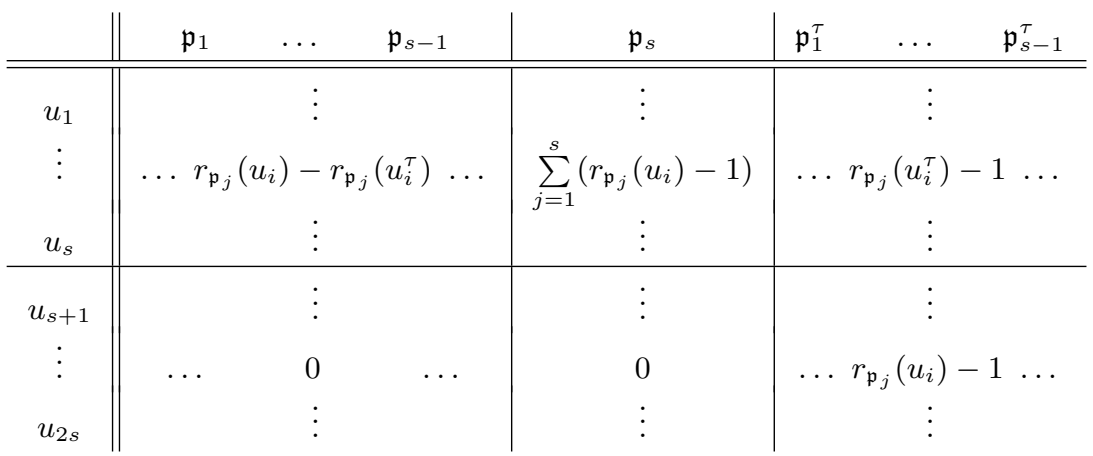

We need to compute the determinant of this matrix if one row is removed. If the removed row is in the upper half of the matrix, then this $(2 s-1) \times(2 s-1)$ matrix contains an $s \times s$ zero submatrix and so its determinant is zero. But if we remove one row in the lower half of the matrix, say row number $s+k$, then the remaining $(2 s-1) \times(2 s-1)$ matrix contains an $(s-1) \times s$ zero submatrix and its determinant is the product of the determinant $D$ of the upper left $s \times s$ submatrix and the determinant $D_{k}$ of the lower right $(s-1) \times(s-1)$ submatrix. Let us compute $D$. We multiply the $s$ th column by 2 and then we subtract the first $s-1$ columns from the $s$ th one. Then the $i$ th entry of the $s$ th column is

$$
\begin{aligned}
2 \sum_{j=1}^{s}\left(r_{\mathfrak{p}_{j}}\left(u_{i}\right)-1\right)-\sum_{j=1}^{s-1}\left(r_{\mathfrak{p}_{j}}\left(u_{i}\right)-r_{\mathfrak{p}_{j}}\left(u_{i}^{\tau}\right)\right) \\
=r_{\mathfrak{p}_{s}}\left(u_{i}\right)-r_{\mathfrak{p}_{s}}\left(u_{i}^{\tau}\right)+\sum_{j=1}^{s}\left(\left(r_{\mathfrak{p}_{j}}\left(u_{i}\right)-1\right)+\left(r_{\mathfrak{p}_{j}^{\tau}}\left(u_{i}\right)-1\right)\right) \\
\quad \equiv r_{\mathfrak{p}_{s}}\left(u_{i}\right)-r_{\mathfrak{p}_{s}}\left(u_{i}^{\tau}\right)
\end{aligned}
$$

modulo $I_{G}^{2}$. Therefore $D$ is congruent to $\frac{1}{2} \operatorname{det}\left(r_{\mathfrak{p}_{j}}\left(u_{i}^{1-\tau}\right)-1\right)_{1 \leq i, j \leq s}=$ $\frac{1}{2} \operatorname{Reg}_{K, S}^{-}$. Since $p_{j}$ splits in $F / \mathbb{Q}$, we have $F_{\mathfrak{p}_{j}}=\mathbb{Q}_{p_{j}}$ and similarly the completions of $K^{+}$and of $K$ coincide, so $r_{\mathfrak{p}_{j}}\left(u_{i}\right)=r_{p_{j}}\left(u_{i}\right)$ and we obtain

$$
D_{k}=\operatorname{Reg}_{K^{+} / \mathbb{Q}, S}\left(u_{s+1} \wedge \cdots \wedge u_{s+k-1} \wedge u_{s+k+1} \wedge \cdots \wedge u_{2 s}\right) .
$$

From the definition of $\varphi^{1}$ we finally obtain

$$
\begin{aligned}
\operatorname{Reg}_{K / F, S}\left(\varphi ^ { 1 } \left(u_{1}\right.\right. & \left.\left.\wedge \cdots \wedge u_{2 s}\right)\right) \\
= & (-1)^{s} \cdot \frac{1}{2} \operatorname{Reg}_{K, S}^{-} \cdot \operatorname{Reg}_{K^{+} / \mathbb{Q}, S}\left(\varphi^{1}\left(u_{s+1} \wedge \cdots \wedge u_{2 s}\right)\right) .
\end{aligned}
$$

The factor $(-1)^{s}$ comes from the alternating signs in the sum that defines $\varphi^{1}$ applied to an $s-1$-fold wedge, and to an $2 s-1$-fold wedge: summand number $s+k$ on the left corresponds to the $k$ th summand on the right.

This argument can be exploited further. Let us return to the part of the argument immediately preceding Proposition 2.4. We let $c(\varphi)=\operatorname{Reg}_{K^{+} / \mathbb{Q}, S}^{\varphi}$. 
If we assume that we can find at least one $\varphi$ such that the multiplication map

$$
-c(\varphi): I_{G}^{s} / I_{G}^{s+1} \rightarrow I_{G}^{2 s-1} / I_{G}^{2 s}
$$

is injective, then the same calculation works backwards. More precisely, we have $\widetilde{\Theta}_{K} \cdot \varphi\left(\eta_{K^{+} / \mathbb{Q}, S}\right)=\widetilde{\Theta}_{K} \cdot\left((-1)^{s+1} \frac{1}{2} c(\varphi)\right)$ because of $(\mathrm{B})$ for $K^{+} / \mathbb{Q}$; and we have $-\widetilde{\Theta}_{K} \cdot \varphi\left(\eta_{K^{+} / \mathbb{Q}, S}\right)=\varphi\left(\eta_{K / F, S}\right)=(-1)^{2 s+1} \frac{h_{F, S}}{w_{F}} \operatorname{Reg}_{K / F, S}^{\varphi}$ by virtue of (B) for $K / F$, and the last term can again be factored as $(-1)^{s+1} \frac{h_{F, S}}{2 w_{F}} \operatorname{Reg}_{K, S}^{-} c(\varphi)$. Thus under our assumption that multiplication by $c(\varphi)$ is injective, we may simplify by $c(\varphi)$; the result is (MC).

It remains to see just when such a $\varphi$ can be found.

Let us assume that $K^{+} / \mathbb{Q}$ is cyclic (hence cyclic of order $l$ ). Let $\sigma$ be a fixed generator of $G$. The genus field of $K^{+}$is the compositum $K_{1} \cdots K_{s}$ with $K_{i}$ the abelian field of conductor $p_{i}$ and degree $l$. We also fix a generator $\sigma_{i}$ of $G_{i}=\operatorname{Gal}\left(K_{i} / \mathbb{Q}\right)$ for each $i$, by the prescription that the extension of $\sigma_{i}$ by identity on the other $K_{j}$ restricts to $\sigma$ on $K$. We define an $s \times s$ matrix $A=\left(a_{i j}\right)$ by the properties that it has zero row sums and for $i \neq j, a_{i j}$ is an integer such that $\sigma_{j}^{a_{i j}}$ equals the Frobenius of $p_{i}$ in $K_{j}$. Then up to sign $\operatorname{Reg}_{K^{+} / \mathbb{Q}, S}\left(p_{1} \wedge \cdots \wedge p_{i-1} \wedge p_{i+1} \wedge \cdots \wedge p_{s}\right)$ equals $A_{i}(\sigma-1)^{s-1}$ modulo $(\sigma-1)^{s}$ where $A_{i}$ is the $(i, i)$-minor of $A$; see [6, Proposition 5.6]. (Hayward does specify the sign, but we do not need it.) Note that $A$ and $A_{i}$ are only uniquely determined modulo $l$. We now claim:

TheOREM 2.5. If $K^{+} / \mathbb{Q}$ is cyclic, $l$ does not divide $h_{F}$, and at least one of the minors $A_{1}, \ldots, A_{s}$ is not zero modulo $l$, then the validity of (B) for $K^{+} / \mathbb{Q}$ and for $K / F$ taken together imply the Minus Conjecture (MC) for $K$. Therefore (MC) holds in this situation as a consequence of Theorems 1.1 and 1.2 .

Proof. Assume that $A_{i}$ is not zero modulo $l$. Either by the theory of Gorenstein rings or by using the isomorphism

$$
\operatorname{Hom}_{\mathbb{Z}[G]}(X, \mathbb{Z}[G]) \ni \varphi \mapsto \varphi^{1} \in \operatorname{Hom}_{\mathbb{Z}}(X, \mathbb{Z}),
$$

where $\varphi^{1}(x)$ is the coefficient of the identity element in $\varphi(x)$, one shows that there exists $\varphi \in \operatorname{Hom}_{\mathbb{Z}[G]}(X, \mathbb{Z}[G])$ with $\varphi^{1}\left(p_{i^{\prime}}\right)=1$ if $i^{\prime}=i$ and 0 else. (For the existence of such a $\varphi$ one has to use the fact that the quotient of $U_{S}(F) / U_{S}(F)_{\text {tor }}$ modulo the subgroup spanned by $p_{1}, \ldots, p_{s}$ has no torsion. Note also that the present definition of $\varphi^{1}$ extends the previous definition.) Then $\operatorname{Reg}_{K^{+} / \mathbb{Q}, S}$ is up to sign just $\operatorname{Reg}_{K^{+} / \mathbb{Q}, S}\left(p_{1} \wedge \cdots \wedge p_{i-1} \wedge p_{i+1} \wedge \cdots \wedge p_{s}\right)$. Because of our assumption, this has the form " $l$-unit times $(\sigma-1)^{s-1}$ ". Since for all $t>0, I^{t} / I^{t+1}$ is cyclic of order $l$, generated by $(\sigma-1)^{t}$, we conclude that multiplication with $c(\varphi)$ is injective (as explained above) for this choice of $\varphi$. 
An aside remark: From a heuristic viewpoint, i.e., if we regard $A$ as a random matrix with zero row sums, it is highly probable that at least one $A_{i}$ is nonzero. More precisely, there are $l^{n(n-1)}$ matrices in $(\mathbb{Z} / l)^{n, n}$ with vanishing row sums, and one can show that exactly

$$
l^{n(n-1)}\left(1-l^{-2}\right)\left(1-l^{-3}\right) \cdots\left(1-l^{-n}\right)
$$

among them have the maximal possible rank $n-1$. By comparison, there are $l^{n^{2}}$ matrices in $(\mathbb{Z} / l)^{n, n}$, and exactly $l^{n^{2}}\left(1-l^{-1}\right)\left(1-l^{-2}\right)\left(1-l^{-3}\right) \cdots\left(1-l^{-n}\right)$ among them are nonsingular; this is a visibly lower proportion.

We ran extensive tests to check on this estimate, only a small part of which will be mentioned here. We let $A$ be the Frobenius matrix attached to a cyclic degree $l$ field $K^{+}$with $s$ ramified primes $p_{1}, \ldots, p_{s}$, all congruent to 1 modulo the prime $l$, and we calculated the rank of $A$ for all such fields with given $s$ and $l$, under the restriction that all $p_{i}<1000$. For $s=3$ and $l=3$, the observed and estimated ratios of fields with $A$ of maximal rank $s-1$ were 0.858024 and 0.855967 respectively (a difference of about 0.2 percent). There were about 330000 fields. For $s=5$ and $l=19$, the observed and estimated ratios were 0.997086 and 0.997076 , that is, very close indeed. There were about 2.2 million fields. For the case $s=3, l=19$, the range of fields was extended further to $p_{i}<10000$, with the result that the observed ratio was still closer to the estimate than it was for $p_{i}<1000$.

If $s=2$ the condition on minors simplifies a lot, so Theorem 2.5 reads as

Corollary 2.6. Let $K^{+} / \mathbb{Q}$ be cyclic, and assume $s=2$ and $l \nmid h_{F}$. If at least one of $p_{1}, p_{2}$ is not an lth power residue modulo the other one, then the Minus Conjecture (MC) holds for $K$.

The hypothesis of Corollary 2.6 is satisfied for instance if $l=3, p_{1}=13$, $p_{2}=37$. It is not satisfied for instance if $l=3, p_{1}=13, p_{2}=229$. It is routine to show that there are (for every $l$ ) infinitely many pairs $p_{1}, p_{2}$ for which the hypothesis is not satisfied, and one even may fix one of the $p_{i}$.

The main aim of this paper is to give a proof of the Minus Conjecture in the case not covered by the previous corollary, so (in our opinion) in the really hard case. Consider the following

Assumption A. $s=2$ and $p_{2}$ is an $l$ th power modulo $p_{1}$.

Our main result will be that the Minus Conjecture is true under Assumption A. As things stand, we can only do it if $l \nmid h_{F}$. We repeat our other hypotheses: $l$ is an odd prime which does not divide the conductor $f$ of $F$; $K=F K^{+}$, and $K^{+} / \mathbb{Q}$ is $l$-elementary abelian, tamely ramified exactly at $p_{1}$ and $p_{2}$ which both split in $F$. By Corollary 2.6, the Minus Conjecture is then true for $s=2$ without any restriction on $p_{1}$ and $p_{2}$, at least in the cyclic case. 
We first deal with the case $s=1$. Actually, this already follows from Theorem 2.5, since the condition on the matrix minors is trivially true for $s=1$ : the determinant of an empty matrix is 1 . But the proof of Theorem 2.5 heavily relies on Hayward's as yet unpublished thesis, because the sign in the conjecture is crucial. So we give a complete argument here, also as a preparation for dealing with $s=2$. We think that there is some explicit arithmetic meaning behind this; see Theorem 3.4 below. We mention right here that this theorem has the following nontrivial congruence as a special case $f=4$ :

$$
\left(\frac{p-1}{4}\right) !^{-8} \equiv \prod_{\substack{a \bmod ^{\times} 4 p \\ a \equiv 1(\bmod 4)}} a^{2 a}(\bmod p)
$$

for all primes $p \equiv 1(\bmod 4)$. It is actually possible here to divide the exponents by 2 and to remove the sign ambiguity. V. Trnková (work in progress) proved that the sign is a minus for all $p$. The authors do not know whether this could be done in Theorem 3.4.

3. The case $s=1$ of the Minus Conjecture. We start with an imaginary quadratic field $F$ of conductor $f$, class number $h_{F}$ and unit-root number $w_{F}$. We let $S$ be the singleton set $\{p\}$, with $p \equiv 1$ modulo $l$ and $p$ split in $F$, as usual. We assume $l \nmid f$, which implies $l \nmid w_{F}$. Using the canonical isomorphism

$$
\iota: I_{G} / I_{G}^{2} \rightarrow G, \quad \overline{\sigma-1} \mapsto \sigma, \quad \sigma \in G,
$$

we can rewrite the Minus Conjecture with $s=1$ as the following statement:

$$
\iota\left(-w_{F} \widetilde{\Theta}_{K}\right)=r_{\mathfrak{p}}\left(u^{1-\tau}\right)^{h_{F, S}} \in G .
$$

We recall the notations: $\mathfrak{p}$ is a chosen prime of $F$ over $p$, and $u^{1-\tau}$ is a suitably chosen generator of $\left(U_{S}(F) / U_{S}(F)_{\text {tor }}\right)^{1-\tau}$. We need to be more precise however. The order of $[\mathfrak{p}]$ in $\mathrm{Cl}_{F}=\mathrm{Cl}_{F}^{-}$is $h_{F} / h_{F, S}$; let $u$ be a generator of $\mathfrak{p}^{h_{F} / h_{F, S}}$. Then $u, p$ are a $\mathbb{Z}$-basis of $U_{S}(F) / U_{S}(F)_{\text {tor }}$, and the basis $u^{1-\tau}$ satisfies the sign rule explained in the statement of the conjecture in $\S 1$. Inserting this in the above formula we obtain the following version:

$$
\iota\left(-w_{F} \widetilde{\Theta}_{K}\right)=r_{\mathfrak{p}}\left(\psi^{1-\tau}\right) \in G,
$$

where $\psi$ is a generator of $\mathfrak{p}^{h_{F}}$.

Let $t$ be the order of $p$ modulo $f$ and $q=p^{t}$. We consider a standard Gauss sum $g_{0}=g\left(\omega^{-(q-1) / f}, \eta\right) \in \mathbb{Q}\left(\zeta_{f}, \zeta_{p}\right)$, where $\omega$ is the Teichmüller character associated to a prime $\mathfrak{P}$ over $\mathfrak{p}$ in $\mathbb{Q}\left(\zeta_{q-1}\right)$, and $\eta$ the standard additive character. Let $D$ be the decomposition field for $p$ in $\mathbb{Q}\left(\zeta_{f}\right) / \mathbb{Q}$, so $F \subseteq D \subseteq \mathbb{Q}\left(\zeta_{f}\right)$ and $\operatorname{Gal}\left(\mathbb{Q}\left(\zeta_{f}\right) / D\right)=\left\langle\sigma_{p}\right\rangle$. Let $g$ be obtained from $g_{0}^{f}$ (which lies in $D$-see [12, Lemmas 6.4 and 6.5]) by taking the norm from $D$ to $F$. 
Lemma 3.1. There is an explicit p-power $p^{n}$ such that

$$
g^{2} p^{n} \mathcal{O}_{F}=\mathfrak{p}^{-2(1-\tau) f h_{F} / w_{F}},
$$

and consequently $g^{2}$ equals $\psi^{-2(1-\tau) f / w_{F}}$ up to a power of $p$ and a root-ofunity factor in $F$.

Proof. The primes above $p$ split in $D / F$ and are inert in $\mathbb{Q}\left(\zeta_{f}\right) / D$. From this one finds that $g \mathcal{O}_{F}=\mathfrak{p}^{\beta}$, where $\beta$ is the image of the Stickelberger element without denominator $f \Theta_{f}=\sum_{a \bmod ^{\times}} a \sigma_{a}^{-1}$ in $\mathbb{Z}[\tau]$. We exponentiate with $1-\tau$. Since $g^{1+\tau}$ is a $p$-power, this changes $g$ to $g^{2}$ times a $p$-power (always modulo roots of unity). On the right hand side we get $\mathfrak{p}^{(1-\tau) \beta}$, and $(1-\tau) \beta=(1-\tau) \chi(\beta)$ with $\chi$ the quadratic character of $F$; finally $\chi(\beta)=f B_{1, \chi}=-2 f h_{F} / w_{F}$ by [12, Theorem 4.17]. This gives the first formula in the lemma, and the second statement is a direct consequence.

The next step is now (and this will reappear later) to consider the $p$-adic leading term $T(g)$ of $g$. This is defined as follows: identify $F_{\mathfrak{p}}^{\times}$with $\mathbb{Q}_{p}^{\times}$, write $g=p^{z} h$ with $z \in \mathbb{Z}$ and $h \in \mathbb{Z}_{p}^{\times}$, and let $T(g)$ be the image of $h$ in $(\mathbb{Z} / p \mathbb{Z})^{\times}$. For $0 \leq a<q-1=p^{t}-1$ we write out $a p$-adically as $a=a_{0}+a_{1} p+\cdots+a_{t-1} p^{t-1}$ with digits $a_{i}$ in the interval $[0, p-1]$, and we put

$$
a ! !=a_{0} ! a_{1} ! \cdots a_{t-1} ! \quad \text { and } \quad s(a)=a_{0}+a_{1}+\cdots+a_{t-1} .
$$

From [12, Remark on p. 97], we obtain the following congruence for any $a \bmod ^{\times} f$ :

$$
g_{0}^{\sigma_{a}}\left(\zeta_{p}-1\right)^{-s(a(q-1) / f)} \equiv(a(q-1) / f) ! !^{-1}\left(\bmod \mathfrak{P}^{\prime}\right),
$$

where $\mathfrak{P}^{\prime}$ is the prime of $\mathbb{Q}\left(\zeta_{q-1}, \zeta_{p}\right)$ above $\mathfrak{P}$. As $f s(a(q-1) / f) \equiv a(q-1) \equiv$ $0(\bmod p-1)$ and $\left(\zeta_{p}-1\right)^{p-1}(-p)^{-1} \equiv 1\left(\bmod \mathfrak{P}^{\prime}\right)$, we obtain by means of a well-known computation of $p$-adic digits

$$
\left.g_{0}^{f \sigma_{a}}(-p)^{-f s(a(q-1) / f) /(p-1)} \equiv \prod_{i=0}^{t-1}\left(p\left\langle\frac{\left\langle a p^{i} / f\right\rangle(q-1)}{p}\right\rangle\right)\right)^{-f}\left(\bmod \mathfrak{P}^{\prime}\right),
$$

where angular brackets mean the fractional part of a rational number.

Let $\chi$ be the nontrivial Dirichlet character attached to $F$. Recalling that $\chi(p)=1$ by hypothesis, we see that if $\sigma_{a}$ runs over $\operatorname{Gal}(D / F)$ then $f\left\langle a p^{i} / f\right\rangle$ runs over all $c \bmod ^{\times} f, \chi(c)=1$, and so

$$
T(g)= \pm \prod_{\substack{c \bmod ^{\times} f \\ \chi(c)=1}}(p\langle c(q-1) /(p f)\rangle) !^{-f} .
$$

From this formula and Lemma 3.1 we obtain (writing $\sigma(a)$ instead of $\sigma_{a}$ ): 
LEMMA 3.2.

$$
r_{\mathfrak{p}}\left(\psi^{(1-\tau)}\right)=\sigma\left(\prod_{\substack{c \bmod ^{\times} f \\ \chi(c)=1}}(p\langle c(q-1) /(p f)\rangle) !\right)^{-w_{F}} .
$$

(A comment on signs: Both Lemma 3.1 and the preceding formula for $T(g)$ have a minus sign in their exponent; these cancel on putting things together; however a new exponent -1 arises, since the local Artin map sends $a$ to $\sigma_{a}^{-1}$.)

The next step is to calculate with the element $\widetilde{\Theta}_{K}$. Recall $K$ is the compositum of $F$ and the degree $l$ field of conductor $p$, and $(1-\tau) \widetilde{\Theta}_{K}$ equals $\Theta_{K}$ as defined in $\S 1$; by the way, $\Theta_{K}$ just differs from the "standard" Stickelberger element attached to $K$ by a multiple of the norm element.

LEMMA 3.3.

$$
\iota\left(-f \widetilde{\Theta}_{K}\right)=\prod_{\substack{a \bmod ^{\times} p f \\ \chi(a)=1}} \sigma_{a}^{a} \in G
$$

Proof. As $\chi(p)=1$, the Frobenius of $p$ on $F$ is trivial and so $\widetilde{\Theta}_{K}$ belongs to $I_{G}$. This gives

$$
2 p f \widetilde{\Theta}_{K}=\sum_{\substack{a \bmod ^{\times} p f \\ \chi(a)=1}}(2 a-p f) \sigma_{a}^{-1}=\sum_{\substack{a \bmod ^{\times} p f \\ \chi(a)=1}}(2 a-p f)\left(\sigma_{a}^{-1}-1\right)
$$

and the lemma follows using the fact that $\prod_{a} \sigma_{a}=1 \in G$.

Our task is to show formula (1), which is, by means of Lemmas 3.2 and 3.3, equivalent to

$$
\sigma\left(\prod_{\substack{c \bmod ^{\times} f \\ \chi(c)=1}}\left(p\left\langle\frac{c(q-1)}{p f}\right\rangle\right) !\right)^{-f}=\prod_{\substack{a \bmod ^{\times} p f \\ \chi(a)=1}} \sigma_{a}^{a} \in G,
$$

and this in turn is equivalent to

$$
\left.\prod_{\substack{c \bmod ^{\times} f \\ \chi(c)=1}}\left(p\left\langle\frac{c(q-1)}{p f}\right\rangle\right)\right)^{-f} \equiv \prod_{\substack{a \bmod ^{\times} p f \\ \chi(a)=1}} a^{a} \in(\mathbb{Z} / p \mathbb{Z})^{\times},
$$

where $\equiv_{l}$ means equality up to a factor which is an $l$ th power. In fact, we will prove the above formula up to 4 -torsion in the multiplicative group $(\mathbb{Z} / p \mathbb{Z})^{\times}$. We will prove the following congruence modulo $p$ (where one can even show that the sign is always " + " if $f$ is even): 
THEOREM 3.4.

$$
\left.\prod_{\substack{c \bmod ^{\times} f \\ \chi(c)=1}}\left(p\left\langle\frac{c(q-1)}{p f}\right\rangle\right)\right)^{-2 f} \equiv \pm \prod_{\substack{a \bmod ^{\times} p f \\ \chi(a)=1}} a^{2 a}(\bmod p) .
$$

We shall see that Theorem 3.4 can be obtained as a consequence of the still sharper results that follow, namely Theorems 3.5 and 3.8. The integers $v(c)$ are defined later on.

Theorem 3.5. For all $0<c<f$ with $\chi(c)=1$ we have

$$
\left(p\left\langle\frac{c(q-1)}{p f}\right\rangle\right) !^{-2 f} \equiv \pm f^{-f v(c)} \prod_{\substack{a \bmod ^{\times} p f \\ a \equiv c(\bmod f)}} a^{2 a}(\bmod p) .
$$

Proof. Let $x_{c}$ denote the product on the right hand side. Let $y_{c}$ denote a similar product with $p$ being replaced by $q=p^{t}$, namely

$$
y_{c}=\prod_{\substack{a \bmod _{a} \times f \\ a \equiv c(\bmod f)}} a^{2 a} .
$$

LEMMA 3.6. $x_{c} \equiv y_{c}(\bmod p)$.

Proof. We fix $a \in\{1, \ldots, f p\}$ coprime to $f p$. We can write down the set of all $\alpha \in\{1, \ldots, f q\}$ coprime to $f q$ that map to $a$ modulo $f p$ : this is simply the set $I=\left\{a+i p f \mid i=0, \ldots, p^{t-1}-1\right\}$. From this we find

$$
\prod_{\alpha \bmod _{\alpha \rightarrow a}{ }^{\times}} \alpha^{2 \alpha} \equiv a^{2 s}(\bmod p),
$$

where $s$ is the sum of all elements in $I$; it equals $p^{t-1} a+\frac{1}{2} p^{t-1}\left(p^{t-1}-1\right) p f$. In particular $2 s$ is congruent to $2 a$ modulo $p-1$; hence $a^{2 s} \equiv a^{2 a}(\bmod p)$. Since this argument works for all choices of $a$, the lemma follows.

We continue with the proof of Theorem 3.5.

The next step is to calculate $y_{c}$ by a certain trick (symmetry). We define

$$
I_{c}=\{a=1, \ldots, f q-1 \mid(a, f q)=1 ; a \equiv c(\bmod f)\} .
$$

Then the set $I_{c}$ carries an involution $\varepsilon$, which induces identity modulo $f$ and multiplication by -1 modulo $q$. In more explicit terms, using the least nonnegative residue $j(c)=\left\langle\frac{2 c}{f}\right\rangle \cdot f$ of $2 c$ modulo $f$ :

$$
\varepsilon(a)= \begin{cases}-a+j(c) q, & a<j(c) q, \\ -a+j(c) q+f q, & a>j(c) q .\end{cases}
$$

It is easily checked that $\prod_{a \in I_{c}} a$ maps to -1 modulo $p$ (Wilson's theorem). We put $I_{c}^{\prime}=\left\{a \in I_{c} \mid a>j(c) q\right\}$ (the set corresponding to the second 
case in the above description of $\varepsilon$ ) and we calculate $\bmod p$ :

$$
\begin{aligned}
y_{c} & \equiv \prod_{a \in I_{c}} a^{a} \varepsilon(a)^{\varepsilon(a)} \equiv \pm \prod_{a \in I_{c}} a^{a+\varepsilon(a)} \\
& \equiv \pm \prod_{a \in I_{c}^{\prime}} a^{f q} \cdot \prod_{a \in I_{c}} a^{j(c) q} \equiv \pm z_{c}^{f},
\end{aligned}
$$

where we have put $z_{c}=\prod_{a \in I_{c}^{\prime}} a$, and used again $\prod_{a \in I_{c}} a \equiv-1(\bmod p)$.

We need a better description of $I_{c}^{\prime}$. Taking the residue modulo $q$ gives the natural map $\nu: I_{c} \rightarrow\{i=1, \ldots, q-1 \mid(p, i)=1\}$, which is bijective.

LEMMA 3.7.

(a) If $c<f / 2$, then

$$
\nu\left(I_{c}^{\prime}\right)=\{d=1, \ldots, q-1 \mid(p, d)=1 ; c / f<\langle d / f\rangle \leq 1-c / f\} .
$$

(b) If $c>f / 2$, then

$\nu\left(I_{c}^{\prime}\right)=\{d=1, \ldots, q-1 \mid(p, d)=1 ;\langle d / f\rangle \leq \widehat{c} / f$ or $1-\widehat{c} / f<\langle d / f\rangle\}$,

where $\widehat{c}=f-c$. Note that the condition on $d$ is exactly the negation of the condition in (a), with $c$ replaced by $\widehat{c}$.

Proof. Let $d$ in $\{1, \ldots, q-1\}$ be prime to $p$. The preimage $\nu^{-1}(d) \in I_{c}$ has the form $d+i q, 0 \leq i<f$. We can find $i$ by noting that $q \equiv 1(\bmod f)$, so $d+i \equiv c(\bmod f)$, that is, $i$ is the least nonnegative residue of $c-d$ modulo $f$. Moreover $\nu^{-1}(d) \in I_{c}^{\prime}$ iff $i \geq j(c)$.

We now first treat case (a), that is, $c<f / 2$. Then $j(c)=2 c$, and $i \geq j(c)$ happens iff $c-d$ is congruent to $2 c, 2 c+1, \ldots, f-1$ modulo $f$, which translates to $d \equiv c+1, c+2, \ldots, f-c(\bmod f)$.

In case (b) we have $c>f / 2$, and $j(c)=2 c-f$. Then $i \geq j(c)$ iff $c-d$ is congruent to $2 c-f, 2 c-f+1, \ldots, f-1$ modulo $f$; this happens iff $d \equiv c+1, c+2, \ldots, f-1,0,1, \ldots, f-c(\bmod f)$. The latter condition translates to: either $d \equiv 0, \ldots, \widehat{c}(\bmod f)$ or $d \equiv f-\widehat{c}+1, \ldots, f-1(\bmod f)$. This proves the lemma.

We turn to the final part of the proof of Theorem 3.5 now, which consists in an explicit calculation of $z_{c}$.

We first assume $c<f / 2$ and put $u(c)=\left|I_{c}^{\prime}\right|$. The last lemma gives

$$
z_{c} \equiv \prod_{i=c+1}^{f-c} \prod_{\substack{d \bmod ^{\times} q \\ d \equiv i(\bmod f)}} d=f^{u(c)} \prod_{i=c+1}^{f-c} \prod_{\substack{d \bmod ^{\times} q \\ d \equiv i(\bmod f)}} \frac{d}{f}(\bmod p) .
$$


The inner product can be simplified using the $p$-adic Gamma function:

$$
\begin{aligned}
\prod_{\substack{d \bmod _{d} q \\
d \equiv i(\bmod f)}} \frac{d}{f} & =\prod_{j=1}^{(q-1) / f}-\frac{\Gamma_{p}((i+j f) / f)}{\Gamma_{p}((i-f+j f) / f)}= \pm \frac{\Gamma_{p}((i+q-1) / f)}{\Gamma_{p}(i / f)} \\
& \equiv \pm \frac{\Gamma_{p}((i-1) / f)}{\Gamma_{p}(i / f)}(\bmod p)
\end{aligned}
$$

as $\Gamma_{p}$ preserves congruences modulo $p$. Therefore

$$
z_{c} \equiv \pm f^{u(c)} \prod_{i=c+1}^{f-c} \frac{\Gamma_{p}((i-1) / f)}{\Gamma_{p}(i / f)}= \pm f^{u(c)} \frac{\Gamma_{p}(c / f)}{\Gamma_{p}((f-c) / f)}(\bmod p) .
$$

Lemma 3.7 gives

$$
z_{c} \cdot z_{f-c} \equiv \prod_{d \bmod ^{\times} q} d \equiv-1(\bmod p)
$$

and so

$$
z_{f-c} \equiv-z_{c}^{-1} \equiv \pm f^{-u(c)} \frac{\Gamma_{p}((f-c) / f)}{\Gamma_{p}(c / f)}(\bmod p) .
$$

Therefore we no longer have to distinguish whether $c<f / 2$ or not. Setting $v(c)=u(c)$ for $c<f / 2$ and $v(c)=-u(f-c)$ for $c>f / 2$, in both cases we have

$$
z_{c} \equiv \pm f^{v(c)} \frac{\Gamma_{p}(c / f)}{\Gamma_{p}((f-c) / f)}(\bmod p) .
$$

In the next step we use the functional equation:

$$
\begin{aligned}
z_{c} & \equiv \pm f^{v(c)} \Gamma_{p}(1-c / f)^{-2} \equiv \pm f^{v(c)} \Gamma_{p}(1+c(q-1) / f)^{-2} \\
& \equiv \pm f^{v(c)} \Gamma_{p}(1+p\langle c(q-1) /(p f)\rangle)^{-2} \\
& = \pm f^{v(c)}(p\langle c(q-1) /(p f)\rangle) !^{-2}(\bmod p) .
\end{aligned}
$$

It follows that

$$
y_{c} \equiv \pm z_{c}^{f} \equiv \pm f^{f v(c)}(p\langle c(q-1) /(p f)\rangle) !^{-2 f}(\bmod p)
$$

and Theorem 3.5 follows.

It remains to prove the following result which shows that all the $f$-powers which we picked up in Theorem 3.5 to prove Theorem 3.4 do not matter in the end.

Let us define an integer $V$ by

$$
V=f \sum_{\substack{c \bmod ^{\times} f \\ \chi(c)=1}} v(c) .
$$

We then have the following result: 
THEOREM 3.8. There is an equality

$$
V=(p-1) p^{t-1} \frac{2 f h_{F}}{w_{F}}
$$

in particular $V$ is divisible by $p-1$, and so $f^{V} \equiv 1(\bmod p)$.

Proof. We first remark that we did not find a simple argument showing just $(p-1) \mid V$; apparently we have to calculate the exact value and use $w_{F} \mid 2 f$.

We recall, slightly adapting our notation:

$$
v(c)=\epsilon_{c}\left|\left\{d \bmod ^{\times} q \mid \widehat{c} / f<\langle d / f\rangle \leq 1-\widehat{c} / f\right\}\right|,
$$

where $\epsilon_{c}$ is +1 or -1 , and $\widehat{c}$ is either $c$ or $f-c$, both according to whether $c<f / 2$ or $c>f / 2$.

Via the involution $c \mapsto f-c$, the residues $c>f / 2$ with $\chi(c)=1$ correspond bijectively to the residues $c<f / 2$ with $\chi(c)=-1$, because of $\chi(-1)=-1$. Hence we can rewrite the sum as follows:

$$
V=f \sum_{\substack{c \bmod ^{\times} f \\ c<f / 2}}\left(\chi(c) \sum_{\substack{d \bmod ^{\times} q \\ c / f<\langle d / f\rangle \leq 1-c / f}} 1\right)=f \sum_{d \bmod ^{\times} q} \sum_{\substack{c \bmod ^{\times} f \\ c<f / 2 \\ c / f<\langle d / f\rangle \leq 1-c / f}} \chi(c) .
$$

We define $\beta(r)=\sum_{c=1}^{r-1} \chi(c)$ for any positive integer $r$. Then $\beta(r)$ depends only on the residue of $r$ modulo $f$ because $\sum_{c=1}^{f} \chi(c)=0$.

We shall show that the inner sum over $c$ in the last displayed formula equals $\beta(d)$. Let $d^{\prime} / f=\langle d / f\rangle$. If $d^{\prime}<f / 2$ then the inner sum in question is simply $\beta\left(d^{\prime}\right)$. For the complementary case $d^{\prime}>f / 2$, the condition $c<d^{\prime} \leq$ $f-c$ is tantamount to $c \leq f-d^{\prime}$. By the change of variables $c \mapsto f-c$ we obtain

$$
\sum_{c=1}^{f-d^{\prime}} \chi(c)=\sum_{c=d^{\prime}}^{f-1} \chi(f-c)=-\sum_{c=d^{\prime}}^{f-1} \chi(c)=-\left(\beta(f)-\beta\left(d^{\prime}\right)\right)=\beta\left(d^{\prime}\right) .
$$

So we end up with $\beta\left(d^{\prime}\right)=\beta(d)$ in both cases. Therefore

$$
V=f \sum_{d \bmod ^{\times} q} \beta(d)=f\left(V^{\prime}-V^{\prime \prime}\right),
$$

where

$$
V^{\prime}=\sum_{d=1}^{q} \beta(d) \quad \text { and } \quad V^{\prime \prime}=\sum_{d=1}^{q / p} \beta(p d)
$$


The analytic class number formula gives

$$
\begin{aligned}
\sum_{d=1}^{f} \beta(d) & =\sum_{d=1}^{f} \sum_{c=1}^{d-1} \chi(c)=\sum_{c=1}^{f-1} \sum_{d=c+1}^{f} \chi(c)=\sum_{c \bmod ^{\times} f}(f-c) \chi(c) \\
& =-\sum_{c \bmod ^{\times} f} c \chi(c)=\frac{2 f h_{F}}{w_{F}} .
\end{aligned}
$$

From this we get $\left(\right.$ recalling that $\left.q=p^{t} \equiv 1(\bmod f)\right)$

$$
V^{\prime}=\frac{q-1}{f} \sum_{d=1}^{f} \beta(d)=(q-1) \frac{2 h_{F}}{w_{F}} .
$$

This takes care of $V^{\prime}$. We shall concentrate on $V^{\prime \prime}$ now:

$$
V^{\prime \prime}=\sum_{d=1}^{q / p} \beta(p d)=\sum_{d=1}^{q / p} \beta(f\langle p d / f\rangle)=\sum_{d=1}^{q / p} \sum_{c=1}^{f\langle p d / f\rangle-1} \chi(c)=\sum_{c=1}^{f-1} r_{c} \chi(c),
$$

where $r_{c}$ means the number of $d \in\left\{1, \ldots, p^{t-1}\right\}$ satisfying $c<f\langle p d / f\rangle$. So $r_{c}$ is the number of pairs $(d, e)$ with $1 \leq d \leq p^{t-1}$ and $1 \leq e \leq f-c-1$ such that $p d \equiv f-e(\bmod f)$, i.e. $d \equiv-e p^{t-1}(\bmod f)$, which means $f \mid e p^{t-1}+d$. But $e p^{t-1}+d$ runs through $p^{t-1}+1, p^{t-1}+2, \ldots,(f-c) p^{t-1}$ without repetitions. So

$$
\begin{aligned}
r_{c} & =\left[\frac{(f-c) p^{t-1}}{f}\right]-\left[\frac{p^{t-1}}{f}\right]=p^{t-1}-\left[\frac{p^{t-1}}{f}\right]+\left[-\frac{c p^{t-1}}{f}\right] \\
& =p^{t-1}-\left[\frac{p^{t-1}}{f}\right]-1-\left[\frac{c p^{t-1}}{f}\right],
\end{aligned}
$$

where we have used $f \nmid c p^{t-1}$. Thus, considering $\chi$ also as a ring homomorphism $\mathbb{Q}\left[\operatorname{Gal}\left(\mathbb{Q}\left(\zeta_{f}\right) / \mathbb{Q}\right)\right] \rightarrow \mathbb{Q}$, we have

$$
\begin{aligned}
V^{\prime \prime} & =-\sum_{c=1}^{f-1}\left[\frac{c p^{t-1}}{f}\right] \chi(c)=-\chi\left(\sum_{c \bmod ^{\times}}\left[\frac{c p^{t-1}}{f}\right] \sigma_{c}^{-1}\right) \\
& =-\chi\left(\left(p^{t-1}-\sigma_{p^{t-1}}\right) \sum_{c \bmod _{f} \times} \frac{c}{f} \sigma_{c}^{-1}\right)=\left(p^{t-1}-1\right) \frac{2 h_{F}}{w_{F}},
\end{aligned}
$$

because $\chi(p)=1$. This gives $V^{\prime}-V^{\prime \prime}=\left(q-p^{t-1}\right) 2 h_{F} / w_{F}$ and Theorem 3.8 follows.

We now see at once that Theorem 3.4 follows from Theorems 3.5 and 3.8. Since Theorem 3.4 implies formula (1), which in turn is equivalent to the Minus Conjecture, we have proved:

Theorem 3.9. The Minus Conjecture is true in case $s=1$ and $l \nmid f$. (The notation and the setup are explained at the beginning of this section.) 


\section{Transformation of $\widetilde{\Theta}_{K}$ and the Vanishing Order Conjecture.} In this section we allow all imaginary fields $F$ (the conductor is written $f$ ) and all values $s \geq 1$, as in $\S \S 1-2$. We repeat that $l \nmid w_{F}$ and we also suppose until further notice that $K^{+}$equals its own genus field $K_{1} \cdots K_{s}$ (recall $K_{i}$ is the degree $l$ conductor $p_{i}$ field). Recall that all primes $p_{1}, \ldots, p_{s}$ split in $F$.

Before starting the proof of the Minus Conjecture it is necessary to process the quantity $\widetilde{\Theta}_{K}$. As a byproduct we will deduce that it is always in $I_{G}^{s}$ (so conjecture (VOC) holds). The expression we are going to get for $\widetilde{\Theta}_{K}$ looks complicated, but for $s=2$ it will fit quite well into the Minus Conjecture since on the regulator side the same structure will appear.

Some notation is required. For all $T \subseteq\{1, \ldots, s\}$ let $K_{T}$ denote the compositum of all $K_{i}$ with $i \in T$; here $K_{\emptyset}=\mathbb{Q}$. Let $G_{T}=\operatorname{Gal}\left(K_{T} / \mathbb{Q}\right)$, which is always tacitly identified with the product of the $G_{\{i\}}=G_{i}$ for $i \in T$. Note that $K_{\{1, \ldots, s\}}=K^{+}$. We also need matrices of Frobenius symbols, related to the matrix $A$ of $\S 2$ but with entries in $I_{G}$. To begin with, let $\alpha_{i j}$ be the Frobenius of $p_{i}$ in $K_{j}(i \neq j)$. For $V \subsetneq U \subseteq\{1, \ldots, s\}$ define a matrix $\widetilde{M}_{V}^{U}=\left(\widetilde{m}_{i j}^{U}\right)_{i, j \in U-V}$ over $I_{G_{U}}$ as follows: for $i \neq j$, we set $\widetilde{m}_{i j}^{U}=\alpha_{i j}^{-1}-1$. For $i=j$ we set

$$
\widetilde{m}_{i i}^{U}=\prod_{j \in U, j \neq i} \alpha_{i j}-1
$$

It is easy to see that the row sums of each matrix $\widetilde{M}_{\emptyset}^{U}$ are zero modulo $I_{G}^{2}$. Finally, let $\widetilde{A}_{V}^{U}=\operatorname{det} \widetilde{M}_{V}^{U}$. For $V=U$ this has to be interpreted as 1 (the determinant of the empty matrix).

For each $T \subseteq\{1, \ldots, s\}$ we set $m_{T}=\prod_{i \in T} p_{i}$ (this is the conductor of $K_{T}$ ), and we have a Stickelberger element $\Theta_{T}$ attached to $F K_{T}$ the same way as $\Theta_{K}$ was attached to $K$ :

$$
\Theta_{T}=(1-\tau) \widetilde{\Theta}_{T}, \quad \widetilde{\Theta}_{T}=\sum_{\lambda \in G_{T}} a^{T}(\lambda) \lambda^{-1},
$$

with

$$
a^{T}(\lambda)=\sum_{\substack{t \bmod ^{\times} f m_{T} \\ \sigma_{t}\left|F=1 \\ \sigma_{t}\right| K_{T}=\lambda}}\left(\frac{t}{f m_{T}}-\frac{1}{2}\right) .
$$

Next we associate to every $T \neq \emptyset$ a term $\widetilde{R}_{T}$ which will later give a contribution towards the leading term of $\widetilde{\Theta}_{K}$.

Definition. The map $\widetilde{g}_{T}: G_{T} \rightarrow I_{G}^{|T|}$ is given by

$$
\widetilde{g}_{T}\left(\prod_{i \in T} \gamma_{i}\right)=\prod_{i \in T}\left(\gamma_{i}-1\right) \quad\left(\text { where } \gamma_{i} \in G_{i} \text { for all } i \in T\right) \text {. }
$$


Now, for $\emptyset \neq T \subseteq\{1, \ldots, s\}$, let

$$
\widetilde{R}_{T}=\sum_{\lambda \in G_{T}} a^{T}(\lambda) \widetilde{g}_{T}\left(\lambda^{-1}\right) .
$$

If life were simple, $\widetilde{R}_{\{1, \ldots, s\}}$ would be equal to $\widetilde{\Theta}_{K}$. This is not the case. We will see that $\widetilde{R}_{\{1, \ldots, s\}}$ is only the "principal term" in a representation of $\widetilde{\Theta}_{K}$ as a sum with many terms.

Proposition 4.1. For any nonempty subset $U$ of $\{1, \ldots, s\}$ we have

$$
\widetilde{\Theta}_{U} \equiv \sum_{\emptyset \neq T \subseteq U} \widetilde{A}_{T}^{U} \widetilde{R}_{T}\left(\bmod I_{G_{U}}^{|U|+1}\right)
$$

Proof. We have to begin with a lemma.

Lemma 4.2. For any sets $V \subseteq U \subseteq\{1, \ldots, s\}$ and any $\lambda \in G_{U-V}$ we have

$$
\sum_{\mu \in G_{V}} a^{U}(\lambda \mu)=\sum_{J \subseteq V}(-1)^{|J|} a^{U-V}\left(\lambda \prod_{u \in J} \operatorname{Frob}_{U-V}\left(p_{u}\right)^{-1}\right),
$$

where $\operatorname{Frob}_{T}(q)$ means Frobenius of $q$ in $\operatorname{Gal}\left(K_{T} / \mathbb{Q}\right)=G_{T}$.

Proof. It is easy to see that our $\Theta_{U}$ is equal to $e^{-} \theta_{f m_{U}}^{\prime}(-1)$ in the notation of Sinnott (see [10]). Using well-known norm relations (see Lemma 12 in [9] for example) we obtain

$$
\begin{aligned}
\operatorname{res}_{F K_{U} / F K_{U-V}} \Theta_{U}=\Theta_{U-V} \prod_{u \in V}\left(1-\operatorname{Frob}_{U-V}\left(p_{u}\right)^{-1}\right) \\
=(1-\tau) \sum_{\lambda \in G_{U-V}} a^{U-V}(\lambda) \lambda^{-1} \sum_{J \subseteq V} \prod_{u \in J}\left(-\operatorname{Frob}_{U-V}\left(p_{u}\right)^{-1}\right) \\
=(1-\tau) \sum_{\lambda \in G_{U-V}} \lambda^{-1} \sum_{J \subseteq V}(-1)^{|J|} a^{U-V}\left(\lambda \prod_{u \in J} \operatorname{Frob}_{U-V}\left(p_{u}\right)^{-1}\right) .
\end{aligned}
$$

On the other hand, from the fact that we can decompose $G_{U}$ into a direct product $G_{U}=G_{V} \times G_{U-V}$, we obtain

$$
\operatorname{res}_{F K_{U} / F K_{U-V}} \Theta_{U}=(1-\tau) \sum_{\lambda \in G_{U-V}} \sum_{\mu \in G_{V}} a^{U}(\lambda \mu) \lambda^{-1} .
$$

Comparing coefficients gives the lemma.

Continuing with the proof of Proposition 4.1, we note that $l$ does not divide the number of roots of unity in $F K_{\{1, \ldots, s\}}$ (since $l \nmid w_{F}$ ). Proposition 2.1 in [10] implies that $a^{T}(\lambda)$ is $l$-integral for any $T \subseteq\{1, \ldots, s\}$, and so $\widetilde{R}_{T} \in I_{G_{T}}^{|T|}$. We shall use induction with respect to $|U|$.

If $|U|=1$ then from the definition and the fact that the coefficients $a^{U}(\lambda)$ sum to zero (see Lemma 4.2 for $V=U$ ) we find at once that $\widetilde{\Theta}_{U}=\widetilde{R}_{U}$. 
Since we have set $\widetilde{A}_{U}^{U}=1$, the assertion of Proposition 4.1 holds true in this case.

Let us suppose $|U|>1$ and that the assertion has been proved for all smaller nonempty sets. Using again the fact that for any $T \subseteq U$ we can decompose $G_{U}$ into a direct product $G_{U}=G_{T} \times G_{U-T}$, the definition of $\widetilde{g}_{U}$ gives, by multiplying out,

$$
\widetilde{R}_{U}=\sum_{T \subseteq U}(-1)^{|U-T|} \sum_{\lambda \in G_{T}} \sum_{\mu \in G_{U-T}} a^{U}(\lambda \mu) \lambda^{-1} .
$$

Lemma 4.2 implies

$$
\begin{aligned}
\widetilde{R}_{U} & =\sum_{T \subseteq U}(-1)^{|U-T|} \sum_{\lambda \in G_{T}} \lambda^{-1} \sum_{J \subseteq U-T}(-1)^{|J|} a^{T}\left(\lambda \prod_{u \in J} \operatorname{Frob}_{T}\left(p_{u}\right)^{-1}\right) \\
& =\sum_{T \subseteq U} \sum_{\lambda \in G_{T}} \lambda^{-1} \sum_{J \subseteq U-T}(-1)^{|U-T-J|}\left(\prod_{u \in J} \operatorname{Frob}_{T}\left(p_{u}\right)^{-1}\right) a^{T}(\lambda) \\
& =\sum_{T \subseteq U} \sum_{J \subseteq U-T}(-1)^{|U-T-J|}\left(\prod_{u \in J} \operatorname{Frob}_{T}\left(p_{u}\right)^{-1}\right) \widetilde{\Theta}_{T} \\
& =\sum_{T \subseteq U} \widetilde{\Theta}_{T} \prod_{u \in U-T}\left(\operatorname{Frob}_{T}\left(p_{u}\right)^{-1}-1\right) .
\end{aligned}
$$

Using the induction hypothesis we get (the congruence is modulo $I_{U}^{|U|+1}$ )

$$
\begin{aligned}
\widetilde{R}_{U}-\widetilde{\Theta}_{U} & =\sum_{\emptyset \neq J \subseteq U} \widetilde{\Theta}_{U-J} \prod_{u \in J}\left(\operatorname{Frob}_{U-J}\left(p_{u}\right)^{-1}-1\right) \\
& \equiv \sum_{\emptyset \neq J \subseteq U}\left(\prod_{u \in J}\left(\operatorname{Frob}_{U-J}\left(p_{u}\right)^{-1}-1\right)\right) \sum_{\emptyset \neq T \subseteq U-J} \widetilde{A}_{T}^{U-J} \widetilde{R}_{T} \\
& =\sum_{\emptyset \neq T \subsetneq U} \widetilde{R}_{T} \sum_{\emptyset \neq J \subseteq U-T} \widetilde{A}_{T}^{U-J} \prod_{u \in J}\left(\operatorname{Frob}_{U-J}\left(p_{u}\right)^{-1}-1\right) .
\end{aligned}
$$

Since

$$
\operatorname{Frob}_{U-J}\left(p_{u}\right)^{-1}-1=\prod_{j \in U-J} \alpha_{u j}^{-1}-1 \equiv \sum_{j \in U-J}\left(\alpha_{u j}^{-1}-1\right)\left(\bmod I_{U-J}^{2}\right),
$$

we have

$$
\widetilde{R}_{U}-\widetilde{\Theta}_{U} \equiv \sum_{\emptyset \neq T \subsetneq U} \widetilde{R}_{T} \sum_{\emptyset \neq J \subseteq U-T} \widetilde{A}_{T}^{U-J} \prod_{u \in J} \sum_{j \in U-J}\left(\alpha_{u j}^{-1}-1\right)\left(\bmod I_{U}^{|U|+1}\right) .
$$

At this point we need to modify the matrix $\widetilde{M}_{T}^{U-J}$ slightly, in order to work with a matrix that has all row sums equal to zero. Define a new matrix $\bar{M}_{V}^{U}$ for $V \subsetneq U \subseteq\{1, \ldots, s\}$. First, $\bar{M}_{\emptyset}^{U}$ has the same entries as $\widetilde{M}_{\emptyset}^{U}$ off the diagonal, and we fill the diagonal in such a way that all row sums become zero. Second, $\bar{M}_{V}^{U}$ is obtained from $\widetilde{M}_{\emptyset}^{U}$ simply by omitting the rows and columns with indices in $V$. Then $\bar{M}_{V}^{U}$ and $\widetilde{M}_{V}^{U}$ are congruent modulo $I_{U}^{2}$. 
We also let $\bar{A}_{V}^{U}=\operatorname{det} \bar{M}_{V}^{U}$. The right hand side in the last formula will stay the same modulo $I_{U}^{|U|+1}$ if $\widetilde{A}$ is replaced by $\bar{A}$. The proposition will thus be proved if we can show that

$$
\sum_{J \subseteq U-T} \bar{A}_{T}^{U-J} \prod_{u \in J} \sum_{j \in U-J}\left(\alpha_{u j}^{-1}-1\right)=0
$$

for any $\emptyset \neq T \subsetneq U$. To show this, we use the following very convenient theorem of Chaiken and Kleitman [2] on matrices and trees.

Theorem 4.3. Let $M=\left(m_{i j}\right)_{i, j \in\{1, \ldots, n\}}$ be a matrix over any commutative ring with zero row sums. For any forest $L$ on $\{1, \ldots, n\}$ put

$$
M(L)=\prod_{(i \rightarrow j) \text { is an edge in } L}\left(-m_{i j}\right) .
$$

Then for any $T \subseteq\{1, \ldots, n\}$,

$$
\operatorname{det}\left(m_{i j}\right)_{i, j \in\{1, \ldots, n\}-T}=\sum_{L} M(L),
$$

where $L$ runs over the set of all forests on $\{1, \ldots, n\}$ whose set of roots is $T$.

In our case, we fix $J \subseteq U-T$ and obtain

$$
\bar{A}_{T}^{U-J}=\sum_{\substack{L \text { is a forest on } U-J \\ \sqrt{L}=T}} \bar{M}_{\emptyset}^{U-J}(L),
$$

where the sum is taken over all forests on the set of vertices $U-J$ and the set of roots $T$. Since $\bar{M}_{\emptyset}^{U-J}$ and the corresponding submatrix of $\bar{M}_{\emptyset}^{U}$ have the same entries off the diagonal, we get

$$
\bar{A}_{T}^{U-J} \prod_{u \in J} \sum_{j \in U-J}\left(-\left(\alpha_{u j}^{-1}-1\right)\right)=\sum_{\substack{L \text { is a forest on } U \\ \sqrt{L}=T \\ J \subseteq \ell(L)}} \bar{M}_{\emptyset}^{U}(L),
$$

where the sum is taken over all forests $L$ on the set of vertices $U$ and the set of roots $T$, whose set of leaves $\ell(L)$ contains $J$. So the left hand side of (2) equals

$$
\begin{gathered}
\sum_{J \subseteq U-T}(-1)^{|J|} \sum_{\substack{L \text { is a forest on } U \\
\bar{L}=T \\
J \subseteq \ell(L)}} \bar{M}_{\emptyset}^{U}(L) \\
\quad=\sum_{\substack{L \text { is a forest on } U \\
\sqrt{L}=T}} \bar{M}_{\emptyset}^{U}(L) \sum_{J \subseteq(U-T) \cap \ell(L)}(-1)^{|J|}=0, \\
\end{gathered}
$$

because $U-T \neq \emptyset$ and hence also $(U-T) \cap \ell(L) \neq \emptyset$. Proposition 4.1 is now proved. 
Since each term $\widetilde{A}_{T}^{U}$ is the determinant of a matrix of size $|U|-|T|$ with entries in $I_{U}$ and each term $\widetilde{R}_{T}$ is clearly in $I_{T}^{|T|}$, we see at once that $\widetilde{\Theta}_{U}$ is in $I_{U}^{|U|}$. On setting $U=\{1, \ldots, s\}$ we now conclude that (VOC) is true, that is, we have:

THEOREM 4.4. Under the assumptions stated at the beginning of the section, the element $\widetilde{\Theta}_{K}=\widetilde{\Theta}_{\{1, \ldots, s\}}$ lies in $I_{G}^{s}$. In other words, the congruence in $(\mathrm{MC})$ is at least true modulo $I_{G}^{s}$ and just says $0 \equiv 0$.

5. Simplification of the Minus Conjecture for $s=2$. Until Theorem 8.9 at the very end of the paper we assume from now on that $s=2$, $S=\left\{p_{1}, p_{2}\right\}$ and that $K^{+}$is its own genus field, that is, $K^{+}=K_{1} K_{2}$.

In the proof of Proposition 4.1 we showed that $\widetilde{R}_{U}=\widetilde{\Theta}_{U}$ for $|U|=1$, and we write $\widetilde{\Theta}_{i}$ for $\widetilde{\Theta}_{\{i\}}$. We will now spell out the assertion of Proposition 4.1 for the case $s=2$. Let $\alpha_{i j}$ be defined as in $\S 4$, i.e. $\alpha_{i j}$ is the Frobenius of $p_{i}$ in $K_{j}$ for $i \neq j$, and set $\alpha_{11}=\alpha_{12}^{-1}, \alpha_{22}=\alpha_{21}^{-1}$. Then

$$
\widetilde{M}_{\emptyset}^{1,2}=\left(\begin{array}{ll}
\alpha_{11}^{-1}-1 & \alpha_{12}^{-1}-1 \\
\alpha_{21}^{-1}-1 & \alpha_{22}^{-1}-1
\end{array}\right) .
$$

The quantities $\widetilde{A}_{\{i\}}^{\{1,2\}}$ are obtained by deleting the $i$ th row and column from this matrix and taking the determinant $(i=1,2)$. Therefore Proposition 4.1 amounts to

$$
\begin{aligned}
\widetilde{\Theta}_{K} & \equiv \widetilde{R}_{1,2}+\left(\alpha_{11}^{-1}-1\right) \widetilde{R}_{2}+\left(\alpha_{22}^{-1}-1\right) \widetilde{R}_{1} \\
& \equiv \widetilde{R}_{1,2}+\left(\alpha_{11}^{-1}-1\right) \widetilde{\Theta}_{2}+\left(\alpha_{22}^{-1}-1\right) \widetilde{\Theta}_{1}\left(\bmod I_{G}^{3}\right)
\end{aligned}
$$

We now show that the minus-unit regulator has a very similar decomposition. Let $\mathfrak{p}_{1}$ and $\mathfrak{p}_{2}$ be prime ideals in $F$ above $p_{1}$ and $p_{2}$, respectively.

Set

$$
\begin{gathered}
t_{1}=\frac{h_{F}}{h_{F,\left\{p_{1}\right\}}}, \quad t_{2}=\frac{h_{F}}{h_{F,\left\{p_{2}\right\}}}, \quad t_{3}=\frac{h_{F} h_{F, S}}{h_{F,\left\{p_{1}\right\}} h_{F,\left\{p_{2}\right\}}}, \\
t_{1}^{\prime}=\frac{h_{F,\left\{p_{1}\right\}}}{h_{F, S}}=\frac{t_{2}}{t_{3}}, \quad t_{2}^{\prime}=\frac{h_{F,\left\{p_{2}\right\}}}{h_{F, S}}=\frac{t_{1}}{t_{3}} .
\end{gathered}
$$

Then $t_{2}$ is the smallest positive integer such that $\mathfrak{p}_{2}^{t_{2}}=\left(u_{2}\right)$ is principal. Similarly $t_{2}^{\prime}$ is the smallest positive integer for which there is an integer $t$ such that $\mathfrak{p}_{1}^{t_{2}^{\prime}} \mathfrak{p}_{2}^{t}=\left(u_{1}\right)$ is principal. Then $u_{1}, u_{2}, p_{1}, p_{2}$ is a basis of $U_{S}(F) / U_{S}(F)$ tor and $u_{1}^{1-\tau}, u_{2}^{1-\tau}$ is a basis of $\left(U_{S}(F) / U_{S}(F)_{\text {tor }}\right)^{1-\tau}$ and it is well-oriented with respect to $\mathfrak{p}_{1}, \mathfrak{p}_{2}$.

With this choice we get $\operatorname{Reg}_{K, S}^{-}=\operatorname{det}\left(r_{\mathfrak{p}_{j}}\left(u_{i}^{1-\tau}\right)-1\right)_{i, j=1,2}$, with $r_{\mathfrak{p}_{j}}(x)$ denoting the local Artin symbol $\left(x, K_{\mathfrak{p}_{j}} / F_{\mathfrak{p}_{j}}\right)$. Note that (here and else- 
where), by a harmless abuse of notation, this symbol stands for $\left(x, K_{\mathfrak{P}} / F_{\mathfrak{p}_{j}}\right)$ with $\mathfrak{P}$ being a prime above $\mathfrak{p}_{j}$ in $K$.

Let $\beta_{i j}=\left(u_{i}^{1-\tau},\left(F K_{j}\right)_{\mathfrak{p}_{j}} / F_{\mathfrak{p}_{j}}\right) \in G_{j} \subseteq G$, for all $i, j \in\{1,2\}$ (including $i=j)$. Let $\widetilde{M}^{-}=\left(\beta_{i j}-1\right)_{i, j=1,2}$.

Proposition 5.1. The following congruence holds modulo $I_{G}^{2}$ :

$$
\left(r_{\mathfrak{p}_{j}}\left(u_{i}^{1-\tau}\right)-1\right)_{i, j=1,2} \equiv \widetilde{M}^{-}+\left(\begin{array}{cc}
t_{2}^{\prime}\left(\alpha_{11}^{-1}-1\right) & t\left(\alpha_{22}^{-1}-1\right) \\
0 & t_{2}\left(\alpha_{22}^{-1}-1\right)
\end{array}\right) .
$$

Proof. We have

$$
r_{\mathfrak{p}_{1}}\left(u_{1}^{1-\tau}\right)=\left(u_{1}^{1-\tau},\left(F K_{1}\right)_{\mathfrak{p}_{1}} / F_{\mathfrak{p}_{1}}\right)\left(u_{1}^{1-\tau},\left(F K_{2}\right)_{\mathfrak{p}_{1}} / F_{\mathfrak{p}_{1}}\right)=\beta_{11} \alpha_{12}^{t_{2}^{\prime}},
$$

because $\left(F K_{2}\right)_{\mathfrak{p}_{1}} / F_{\mathfrak{p}_{1}}$ is unramified and the $\mathfrak{p}_{1}$-valuation of $u_{1}^{1-\tau}$ is $t_{2}^{\prime}$. Similarly

$$
\begin{aligned}
& r_{\mathfrak{p}_{1}}\left(u_{2}^{1-\tau}\right)=\left(u_{2}^{1-\tau},\left(F K_{1}\right)_{\mathfrak{p}_{1}} / F_{\mathfrak{p}_{1}}\right)\left(u_{2}^{1-\tau},\left(F K_{2}\right)_{\mathfrak{p}_{1}} / F_{\mathfrak{p}_{1}}\right)=\beta_{21}, \\
& r_{\mathfrak{p}_{2}}\left(u_{1}^{1-\tau}\right)=\left(u_{1}^{1-\tau},\left(F K_{1}\right)_{\mathfrak{p}_{2}} / F_{\mathfrak{p}_{2}}\right)\left(u_{1}^{1-\tau},\left(F K_{2}\right)_{\mathfrak{p}_{2}} / F_{\mathfrak{p}_{2}}\right)=\alpha_{21}^{t} \beta_{12}, \\
& r_{\mathfrak{p}_{2}}\left(u_{2}^{1-\tau}\right)=\left(u_{2}^{1-\tau},\left(F K_{1}\right)_{\mathfrak{p}_{2}} / F_{\mathfrak{p}_{2}}\right)\left(u_{2}^{1-\tau},\left(F K_{2}\right)_{\mathfrak{p}_{2}} / F_{\mathfrak{p}_{2}}\right)=\alpha_{21}^{t} \beta_{22} .
\end{aligned}
$$

The proposition follows using the canonical isomorphism $I_{G} / I_{G}^{2} \cong G$.

On taking determinants in Proposition 5.1 we find:

Corollary 5.2. The following congruence holds modulo $I_{G}^{3}$ :

$$
\begin{aligned}
\operatorname{Reg}_{K, S}^{-} \equiv \operatorname{det}\left(\widetilde{M}^{-}\right) & +t_{2}^{\prime}\left(\alpha_{11}^{-1}-1\right)\left(\beta_{22}-1\right)+t_{2}\left(\alpha_{22}^{-1}-1\right)\left(\beta_{11}-1\right) \\
& +t_{2} t_{2}^{\prime}\left(\alpha_{11}^{-1}-1\right)\left(\alpha_{22}^{-1}-1\right)-t\left(\alpha_{22}^{-1}-1\right)\left(\beta_{21}-1\right) .
\end{aligned}
$$

It is easy to see that $u_{2}^{1-\tau}$ is a basis of $\left(U_{p_{2}}(F) / U_{p_{2}}(F)_{\text {tor }}\right)^{1-\tau}$ and so

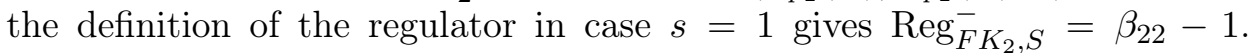
Writing the principal ideal $\mathfrak{p}_{1}^{t_{1}}$ in the form $\mathfrak{p}_{1}^{t_{3} t_{2}^{\prime}}=\left(\left(u_{1}\right) \cdot \mathfrak{p}_{2}^{-t}\right)^{t_{3}}$ shows that $t_{2} \mid t t_{3}$ and $\mathfrak{p}_{1}^{t_{1}}=\left(u_{1}^{t_{3}} u_{2}^{t^{\prime}}\right)$ with $t^{\prime}=-t t_{3} / t_{2}$. Then $\left(u_{1}^{t_{3}} u_{2}^{t^{\prime}}\right)^{1-\tau}$ is a basis of $\left(U_{p_{1}}(F) / U_{p_{1}}(F)_{\text {tor }}\right)^{1-\tau}$ and

$$
\begin{aligned}
\left(\left(u_{1}^{t_{3}} u_{2}^{t^{\prime}}\right)^{1-\tau},\left(F K_{1}\right)_{\mathfrak{p}_{1}} / F_{\mathfrak{p}_{1}}\right) & =\left(u_{1}^{1-\tau},\left(F K_{1}\right)_{\mathfrak{p}_{1}} / F_{\mathfrak{p}_{1}}\right)^{t_{3}}\left(u_{2}^{1-\tau},\left(F K_{1}\right)_{\mathfrak{p}_{1}} / F_{\mathfrak{p}_{1}}\right)^{t^{\prime}} \\
& =\beta_{11}^{t_{3}} \beta_{21}^{t^{\prime}},
\end{aligned}
$$

hence

$$
\operatorname{Reg}_{F K_{1}, S}^{-} \equiv t_{3}\left(\beta_{11}-1\right)-\frac{t t_{3}}{t_{2}}\left(\beta_{21}-1\right)\left(\bmod I_{G_{1}}^{2}\right) .
$$

Therefore substituting $t_{2}\left(\beta_{11}-1\right) \equiv t\left(\beta_{21}-1\right)+\left(t_{2} / t_{3}\right) \operatorname{Reg}_{F K_{1}, S}^{-}$into the 
congruence of Corollary 5.2 gives

$$
\begin{aligned}
\operatorname{Reg}_{K, S}^{-} \equiv \operatorname{det}\left(\widetilde{M}^{-}\right) & +t_{2}^{\prime}\left(\alpha_{11}^{-1}-1\right) \operatorname{Reg}_{F K_{2}, S}^{-} \\
& +\left(\alpha_{22}^{-1}-1\right)\left(t\left(\beta_{21}-1\right)+t_{1}^{\prime} \operatorname{Reg}_{F K_{1}, S}^{-}\right) \\
& +t_{2} t_{2}^{\prime}\left(\alpha_{11}^{-1}-1\right)\left(\alpha_{22}^{-1}-1\right)-t\left(\alpha_{22}^{-1}-1\right)\left(\beta_{21}-1\right) .
\end{aligned}
$$

After rearrangement and simplification this gives

Corollary 5.3. Modulo $I_{G}^{3}$ we have

$$
\begin{aligned}
\operatorname{Reg}_{K, S}^{-} \equiv \operatorname{det}\left(\widetilde{M}^{-}\right) & +\frac{h_{F,\left\{p_{2}\right\}}}{h_{F, S}}\left(\alpha_{11}^{-1}-1\right) \operatorname{Reg}_{F K_{2}, S}^{-} \\
& +\frac{h_{F,\left\{p_{1}\right\}}}{h_{F, S}}\left(\alpha_{22}^{-1}-1\right) \operatorname{Reg}_{F K_{1}, S}^{-} \\
& +\frac{h_{F}}{h_{F, S}}\left(\alpha_{11}^{-1}-1\right)\left(\alpha_{22}^{-1}-1\right) .
\end{aligned}
$$

We now return to formula (3). Since (MC) is proved for $s=1$, we may replace the theta terms by minus regulators as follows:

$$
\begin{aligned}
\widetilde{\Theta}_{K} \equiv & \widetilde{R}_{1,2}-\frac{h_{F,\left\{p_{2}\right\}}}{w_{F}}\left(\alpha_{11}^{-1}-1\right) \operatorname{Reg}_{F K_{2}, S}^{-} \\
& -\frac{h_{F,\left\{p_{1}\right\}}}{w_{F}}\left(\alpha_{22}^{-1}-1\right) \operatorname{Reg}_{F K_{1}, S}^{-}\left(\bmod I_{G}^{3}\right) .
\end{aligned}
$$

On comparing this with Corollary 5.3 we see that $\widetilde{\Theta}_{K} \equiv-\left(h_{F, S} / w_{F}\right) \operatorname{Reg}_{K, S}^{-}$ if and only if

$$
\widetilde{R}_{1,2} \equiv-\frac{h_{F, S}}{w_{F}} \operatorname{det}\left(\widetilde{M}^{-}\right)-\frac{h_{F}}{w_{F}}\left(\alpha_{11}^{-1}-1\right)\left(\alpha_{22}^{-1}-1\right)\left(\bmod I_{G}^{3}\right) .
$$

Under Assumption (A), the automorphism $\alpha_{21}$ and hence also $\alpha_{11}$ is the identity. In any case $l\left(\alpha_{11}^{-1}-1\right)\left(\alpha_{22}^{-1}-1\right) \in I_{G}^{3}$. So we have proved:

Corollary 5.4. Under Assumption (A), or if $l \mid h_{F}$, the Minus Conjecture (MC) for $s=2$ is equivalent to the following congruence:

$$
\widetilde{R}_{1,2} \equiv-\frac{h_{F, S}}{w_{F}} \operatorname{det}\left(\widetilde{M}^{-}\right)\left(\bmod I_{G}^{3}\right)
$$

Actually both sides of this congruence are in the submodule $I_{G_{1}} I_{G_{2}}$ of $I_{G}^{2}$. This will further simplify our task. It is easily verified that the canonical map

$$
I_{G_{1}} / I_{G_{1}}^{2} \otimes_{\mathbb{Z}} I_{G_{2}} / I_{G_{2}}^{2} \rightarrow I_{G}^{2} / I_{G}^{3}
$$

is well-defined and injective. (Indeed, both $I_{G_{1}} / I_{G_{1}}^{2}$ and $I_{G_{2}} / I_{G_{2}}^{2}$ are copies of $\mathbb{Z} / l \mathbb{Z}$, so it suffices to see that the map is not zero.) Putting together the canonical isomorphisms $\iota_{j}: I_{G_{j}} / I_{G_{j}}^{2} \rightarrow G_{j}$, we get a canonical isomorphism

$$
\iota=\iota_{1,2}: I_{G_{1}} / I_{G_{1}}^{2} \otimes_{\mathbb{Z}} I_{G_{2}} / I_{G_{2}}^{2} \rightarrow G_{1} \otimes_{\mathbb{Z}} G_{2} .
$$


It is our intention to consider the formula in Corollary 5.4 above as an equality (to be proven) in $G_{1} \otimes_{\mathbb{Z}} G_{2}$. We want to avoid working with chosen generators of $G_{1}$ and $G_{2}$ as long as possible. In other words, we would like to keep the argument "coordinate-free" as long as feasible. However, a quaint little problem of notation comes up. The groups $G_{j}$ are naturally multiplicative groups, but one should rather work with additively written groups when looking at tensor products over $\mathbb{Z}$. On the other hand, additive notation for $G_{j}$ would look awful (try it!). For lack of a better idea we introduce, for any multiplicative abelian group $\Gamma$, an additive group $\lg \Gamma$, which is just a copy of $\Gamma$. The symbol lg is just formal (reminiscent of a logarithm), and

$$
\lg \Gamma=\{\lg \gamma \mid \gamma \in \Gamma\}, \quad \lg \gamma+\lg \delta=\lg (\gamma \delta) \quad \forall \gamma, \delta \in \Gamma .
$$

Now we put $R_{1,2}=\iota_{1,2}\left(\widetilde{R}_{1,2}\right)$. We note that

$$
\iota_{1,2} \widetilde{g}_{1,2}\left(\gamma_{1} \gamma_{2}\right)=\lg \gamma_{1} \otimes \lg \gamma_{2}
$$

and

$$
\iota_{1,2} \operatorname{det}\left(\widetilde{M}^{-}\right)=\lg \beta_{11} \otimes \lg \beta_{22}-\lg \beta_{21} \otimes \lg \beta_{12} .
$$

Thus,

$$
\begin{aligned}
R_{1,2} & =\sum_{\gamma_{1} \in G_{1}} \sum_{\gamma_{2} \in G_{2}} a^{1,2}\left(\gamma_{1} \gamma_{2}\right) \lg \left(\gamma_{1}^{-1}\right) \otimes \lg \left(\gamma_{2}^{-1}\right) \\
& =\sum_{\gamma_{1} \in G_{1}} \sum_{\gamma_{2} \in G_{2}} a^{1,2}\left(\gamma_{1} \gamma_{2}\right)\left(-\lg \gamma_{1}\right) \otimes\left(-\lg \gamma_{2}\right) \\
& =\sum_{\gamma_{1} \in G_{1}} \sum_{\gamma_{2} \in G_{2}} a^{1,2}\left(\gamma_{1} \gamma_{2}\right) \lg \gamma_{1} \otimes \lg \gamma_{2}
\end{aligned}
$$

and the formula of Corollary 5.4 can be rewritten as follows.

Corollary 5.5. (Assumptions as in Corollary 5.4.) The Minus Conjecture is equivalent to the following equality in $\lg G_{1} \otimes_{\mathbb{Z}} \lg G_{2}$ :

$$
\begin{aligned}
\sum_{\gamma_{1} \in G_{1}} \sum_{\gamma_{2} \in G_{2}} a^{1,2}\left(\gamma_{1} \gamma_{2}\right) \lg \gamma_{1} & \otimes \lg \gamma_{2} \\
= & -\frac{h_{F, S}}{w_{F}}\left(\lg \beta_{11} \otimes \lg \beta_{22}-\lg \beta_{21} \otimes \lg \beta_{12}\right) .
\end{aligned}
$$

6. A formula for $R_{1,2}$. In this section we shall continue to assume $s=2$. For convenience, we will very often write $p$ for $p_{1}$ and $q$ for $p_{2}$. (Thus, $q$ will never denote a $p$-power.) To explain the outcome of this section we need some notation (slightly different from earlier notations): For $u \in(\mathbb{Z} / p \mathbb{Z})^{\times}$let $\sigma_{u} \in G_{1}=\operatorname{Gal}\left(F K_{1} / F\right)$ be given by the condition that $\sigma_{u}$ is the restriction of $\zeta_{p} \mapsto \zeta_{p}^{u}$ on $K_{1}$ and identity on $F$. We define $\tau_{v} \in G_{2}=\operatorname{Gal}\left(F K_{2} / F\right)$ similarly for $v \in(\mathbb{Z} / q \mathbb{Z})^{\times}$. (No relation with $\tau=$ complex conjugation.) 
Recall that $\chi$ is the nontrivial Dirichlet character attached to $F$ and that $f$ is the conductor of $F$ (and so also of $\chi$ ).

THEOREM 6.1. If $\gamma(u)$ stands for $\Gamma_{q}(u /(f p))$, then

$$
R_{1,2}=\sum_{\substack{a \bmod ^{\times} p f \\ \chi(a)=1}} \lg \sigma_{a} \otimes \lg \tau_{\gamma(a)} .
$$

Since this formula is probably not very enlightening at first sight, we give a numerical example. Take $l=3, f=4, p=13$, and omit the $\lg$ symbols for simplicity. Then (the dependence on $q$ is hidden in $\gamma$ ):

$$
\begin{aligned}
R_{1,2}= & \sigma_{5} \otimes \tau_{\gamma(5)}+\sigma_{9} \otimes \tau_{\gamma(9)}+\sigma_{4} \otimes \tau_{\gamma(17)}+\sigma_{8} \otimes \tau_{\gamma(21)} \\
& +\sigma_{12} \otimes \tau_{\gamma(25)}+\sigma_{3} \otimes \tau_{\gamma(29)}+\sigma_{7} \otimes \tau_{\gamma(33)}+\sigma_{11} \otimes \tau_{\gamma(37)} \\
& +\sigma_{2} \otimes \tau_{\gamma(41)}+\sigma_{6} \otimes \tau_{\gamma(45)}+\sigma_{10} \otimes \tau_{\gamma(49)} \in G_{1} \otimes G_{2} \cong \mathbb{Z} / 3 \mathbb{Z} .
\end{aligned}
$$

Note that we omitted $\sigma_{1} \otimes \tau_{\gamma(1)}$ since $\lg \sigma_{1} \otimes \lg \tau_{\gamma(1)}$ is the zero element of $\lg G_{1} \otimes \lg G_{2}$.

Before we enter the proof of Theorem 6.1, let us mention that it arose as the result of at least two successive generalizations, which make it very technical and unfortunately not very enlightening. However, we think we would lose more by abandoning generality than we would gain. The strange-looking Lemma 6.3 (which was also seriously tested by computer, to minimize error probability) has the purpose of eliminating an ungainly obstruction term, which unavoidably appears in the main part of the proof. Proposition 6.2 is just a preparation for this important lemma. It is recommended to skip over Proposition 6.2 and Lemma 6.3 at the first reading (going directly to the not so long proof of Theorem 6.1) and refer back to them later.

For any integer $a$ we define nonnegative integers $r(a)<f p q, h(a)<f p$ by the following conditions:

$$
\begin{aligned}
& r(a) \equiv h(a) \equiv 2 a(\bmod f), \\
& r(a) \equiv h(a) \equiv 0(\bmod p), \\
& r(a) \equiv p f(\bmod q) .
\end{aligned}
$$

Notice that both $r(a)$ and $h(a)$ only depend on $a$ modulo $f$. The following proposition holds true for any odd quadratic Dirichlet character $\chi$ of conductor $f$ and any primes $p, q$ such that $p \neq q$ and $\chi(p)=\chi(q)=1$ :

Proposition 6.2. Let $u=\#\left\{t \bmod ^{\times} f \mid \chi(t)=1,\langle 2 t / f\rangle<1 / q\right\}$ (so in particular $u=0$ for $f \leq 4)$. Let $v=q^{(p-1) / 3}$ if $f=3, v=q^{(p-1) / 2}$ if $f=4$, and $v=(-1)^{u}$ if $f>4$. Then

$$
\prod_{\substack{0<a<r(a) \\ \chi(a)=-1 \\ q \mid a, p \nmid a}} a \equiv v \cdot \prod_{\substack{0<a<p f \\ \chi(a)=1 \\ q \mid a, p \nmid a}} a \cdot \prod_{\substack{h(a)<a<p f \\ \chi(a)=1 \\ p \nmid a}} a(\bmod p) .
$$


Proof. Let $n_{1}, n_{2}, n_{3}$ be the number of factors in the three products above. By means of the substitution $b=a+p q t$, where $t$ is determined by $-p q t \equiv a(\bmod f)$, we obtain

$$
\begin{aligned}
n_{1} & =\sum_{\substack{t \bmod ^{\times} f \\
\chi(t)=1}} \#\{b|p q t<b<r(-p q t)+p q t, q f| b, p \nmid b\} \\
& =\sum_{\substack{t \bmod ^{\times} f \\
\chi(t)=1}}\left(\left[\frac{r(-p q t)+p q t}{q f}\right]-\left[\frac{p q t}{q f}\right]-\left[\frac{r(-p q t)+p q t}{p q f}\right]+\left[\frac{p q t}{p q f}\right]\right) .
\end{aligned}
$$

It is easy to check that $r(-p q t) \equiv-2 p q t+p f(\bmod p q f)$ and so $r(-p q t)=$ $p q f\langle-2 t / f+1 / q\rangle=-2 p q t+p f-p q f[-2 t / f+1 / q]$, which gives

$$
\begin{aligned}
n_{1}= & \sum_{\substack{t \bmod ^{\times} f \\
\chi(t)=1}}\left(\left[-\frac{p t}{f}+\frac{p}{q}-p\left[-\frac{2 t}{f}+\frac{1}{q}\right]\right]-\left[\frac{p t}{f}\right]\right. \\
& \left.\quad-\left[-\frac{t}{f}+\frac{1}{q}-\left[-\frac{2 t}{f}+\frac{1}{q}\right]\right]+\left[\frac{t}{f}\right]\right) \\
\equiv & \sum_{\substack{t \bmod ^{\times} f \\
\chi(t)=1}}\left(\left[-\frac{p t}{f}+\frac{p}{q}\right]-\left[\frac{p t}{f}\right]-\left[-\frac{t}{f}+\frac{1}{q}\right]+\left[\frac{t}{f}\right]\right)(\bmod p-1) .
\end{aligned}
$$

Therefore the first product in (6) is

$$
\begin{aligned}
& \prod_{\substack{0<a<r(a) \\
\chi(a)=-1 \\
q \mid a, p \nmid a}} a \equiv(f q)^{n_{1}} \prod_{\substack{t \bmod ^{\times} \times \\
\chi(t)=1}} \prod_{\substack{p q t<b<r(-p q t)+p q t \\
q f \mid b, p \nmid b}} \frac{b}{f q}(\bmod p) \\
& =(f q)^{n_{1}}(-1)^{c_{1}} \prod_{\substack{t \bmod ^{\times} f \\
\chi(t)=1}} \Gamma_{p}\left(1+\left[-\frac{p t}{f}+\frac{p}{q}\right]-p\left[-\frac{2 t}{f}+\frac{1}{q}\right]\right) \Gamma_{p}\left(1+\left[\frac{p t}{f}\right]\right)^{-1} \\
& \equiv(f q)^{n_{1}}(-1)^{c_{1}} \prod_{\substack{t \bmod ^{\times} f \\
\chi(t)=1}} \Gamma_{p}\left(1+\left[-\frac{p t}{f}+\frac{p}{q}\right]\right) \Gamma_{p}\left(1+\left[\frac{p t}{f}\right]\right)^{-1}(\bmod p),
\end{aligned}
$$

where $c_{1}=\sum_{t \bmod ^{\times}} f, \chi(t)=1([-p t / f+p / q]-p[-2 t / f+1 / q]-[p t / f])$.

The second product in (6) can be treated by means of the substitution $b=a-p q t$, where $t$ is determined by $p q t \equiv a(\bmod f)$. We obtain

$$
n_{2}=\sum_{\substack{t \bmod ^{\times} f \\ \chi(t)=1}} \#\{b|-p q t<b<p f-p q t, q f| b, p \nmid b\}
$$




$$
\begin{aligned}
= & \sum_{\substack{t \bmod ^{\times} f \\
\chi(t)=1}}\left(\left[\frac{p f-p q t}{q f}\right]-\left[\frac{-p q t}{q f}\right]-\left[\frac{p f-p q t}{p q f}\right]+\left[\frac{-p q t}{p q f}\right]\right) \\
= & \sum_{\substack{t \bmod ^{\times} f \\
\chi(t)=1}}\left(\left[-\frac{p t}{f}+\frac{p}{q}\right]-\left[-\frac{p t}{f}\right]-\left[-\frac{t}{f}+\frac{1}{q}\right]+\left[-\frac{t}{f}\right]\right)
\end{aligned}
$$

and

$$
\begin{aligned}
& \prod_{\substack{0<a<p f \\
\chi(a)=1 \\
q \mid a, p \nmid a}} a \equiv(f q)^{n_{2}} \prod_{\substack{t \bmod ^{\times} f \\
\chi(t)=1}} \prod_{\substack{-p q t<b<p f-p q t \\
q f \mid b, p \nmid b}} \frac{b}{f q}(\bmod p) \\
& =(f q)^{n_{2}}(-1)^{c_{2}} \prod_{\substack{t \bmod ^{\times} f \\
\chi(t)=1}} \Gamma_{p}\left(1+\left[-\frac{p t}{f}+\frac{p}{q}\right]\right) \Gamma_{p}\left(1+\left[-\frac{p t}{f}\right]\right)^{-1},
\end{aligned}
$$

where $c_{2}=\sum_{t \bmod ^{\times} f, \chi(t)=1}([-p t / f+p / q]-[-p t / f])$.

Similarly by means of the substitution $b=a-p t$, where $t$ is determined by $p t \equiv a(\bmod f)$, we obtain

$$
\begin{aligned}
n_{3} & =\sum_{\substack{t \bmod ^{\times} f \\
\chi(t)=1}} \#\{b|h(p t)-p t<b<p f-p t, f| b, p \nmid b\} \\
& =\sum_{\substack{t \bmod ^{\times} f \\
\chi(t)=1}}\left(\left[\frac{p f-p t}{f}\right]-\left[\frac{h(p t)-p t}{f}\right]-\left[\frac{p f-p t}{p f}\right]+\left[\frac{h(p t)-p t}{p f}\right]\right) .
\end{aligned}
$$

It is easy to check that $h(p t) \equiv 2 p t(\bmod p f)$ and so $h(p t)=p f\langle 2 t / f\rangle=$ $2 p t-p f[2 t / f]$, which gives

$$
\begin{aligned}
n_{3} & =\sum_{\substack{t \bmod ^{\times} f(t)=1 \\
\chi(t)}}\left(\left[p-\frac{p t}{f}\right]-\left[\frac{p t}{f}-p\left[\frac{2 t}{f}\right]\right]-\left[1-\frac{t}{f}\right]+\left[\frac{t}{f}-\left[\frac{2 t}{f}\right]\right]\right) \\
& \equiv \sum_{\substack{t \bmod ^{\times} f \\
\chi(t)=1}}\left(\left[-\frac{p t}{f}\right]-\left[\frac{p t}{f}\right]-\left[-\frac{t}{f}\right]+\left[\frac{t}{f}\right]\right)(\bmod p-1) .
\end{aligned}
$$

Thus the third product in (6) is

$$
\prod_{\substack{h(a)<a<p f \\ \chi(a)=1 \\ p \nmid a}} a \equiv f^{n_{3}} \prod_{\substack{t \bmod ^{\times} f \\ \chi(t)=1}} \prod_{\substack{h(p t)-p t<b<p f-p t \\ f \mid b, p \nmid b}} \frac{b}{f}(\bmod p)
$$




$$
\begin{gathered}
=f^{n_{3}}(-1)^{c_{3}} \prod_{\substack{t \bmod ^{\times} f \\
\chi(t)=1}} \Gamma_{p}\left(1+p+\left[-\frac{p t}{f}\right]\right) \Gamma_{p}\left(1+\left[\frac{p t}{f}\right]-p\left[\frac{2 t}{f}\right]\right)^{-1} \\
\equiv f^{n_{3}}(-1)^{c_{3}} \prod_{\substack{t \bmod ^{\times} f \\
\chi(t)=1}} \Gamma_{p}\left(1+\left[-\frac{p t}{f}\right]\right) \Gamma_{p}\left(1+\left[\frac{p t}{f}\right]\right)^{-1}(\bmod p),
\end{gathered}
$$

where $c_{3}=\sum_{t \bmod ^{\times} f, \chi(t)=1}(p+[-p t / f]-[p t / f]+p[2 t / f])$.

Putting these results together yields $n_{1}-n_{2}-n_{3} \equiv 0(\bmod p-1)$ and

$$
\prod_{\substack{0<a<r(a) \\
\chi(a)=-1}} a \cdot \prod_{\substack { 0<a<p f \\
\begin{subarray}{c}{0(a)=1 \\
q \mid a, p \nmid a{ 0 < a < p f \\
\begin{subarray} { c } { 0 ( a ) = 1 \\
q | a , p \nmid a } } \\
{q \mid a, p \nmid a}\end{subarray}} a^{-1} \cdot \prod_{\substack{h(a)<a<p f \\
\chi(a)=1 \\
p \nmid a}} a^{-1} \equiv q^{n_{3}}(-1)^{c_{1}-c_{2}-c_{3}}(\bmod p) .
$$

The proposition will be proved if we show that the right hand side is congruent to $v$. If $p=2$ then the parity of $c_{1}-c_{2}-c_{3}$ is not important, while if $p$ is odd then

$$
\begin{aligned}
c_{1}-c_{2}-c_{3} & \equiv \sum_{\substack{t \bmod ^{\times} f \\
\chi(t)=1}}\left(1+\left[\frac{2 t}{f}\right]+\left[-\frac{2 t}{f}+\frac{1}{q}\right]\right) \\
& \equiv \sum_{\substack{t \bmod ^{\times} f \\
\chi(t)=1}}\left(\left[\frac{2 t}{f}\right]-\left[\frac{2 t}{f}-\frac{1}{q}\right]\right) \equiv u(\bmod 2) .
\end{aligned}
$$

We have the following congruence modulo $p-1$ :

$$
\begin{aligned}
n_{3} & \equiv 2 \sum_{\substack{t \bmod ^{\times} f(t)=1 \\
\text { ( }}}\left(\left[\frac{t}{f}\right]-\left[\frac{p t}{f}\right]\right)=2 \sum_{\substack{t \bmod ^{\times} f \\
\chi(t)=1}}\left(\frac{t}{f}-\frac{p t}{f}-\left\langle\frac{t}{f}\right\rangle+\left\langle\frac{p t}{f}\right\rangle\right) \\
& =\frac{2(1-p)}{f} \sum_{\substack{t \bmod ^{\times} f \\
\chi(t)=1}} t=\frac{1-p}{f} \sum_{t \bmod ^{\times} f}(1+\chi(t)) t \\
& =(1-p)\left(\frac{\varphi(f)}{2}-\frac{2 h_{F}}{w_{F}}\right)(\bmod p-1) .
\end{aligned}
$$

If $f=3$ then $n_{3} \equiv(1-p)(1-1 / 3) \equiv \frac{1}{3}(p-1)$, if $f=4$ then $n_{3} \equiv$ $(1-p)(1-1 / 2) \equiv \frac{1}{2}(p-1)$. Finally, if $f>4$ then $n_{3} \equiv 0$. In all cases $q^{n_{3}}(-1)^{c_{1}-c_{2}-c_{3}} \equiv v(\bmod p)$ and the proposition is proved.

LEMMA 6.3. Let $d$ be any integer satisfying $p f d \equiv 1(\bmod q)$. Then the integer 


$$
n=\prod_{\substack{a \bmod ^{\times} p f \\ q \nmid a, \chi(a)=1}} a^{q\langle d a / q\rangle-1}
$$

is an lth power modulo $p$.

Proof. Since $0<f p-h(a)+f p q\langle d h(a) / q\rangle<f p q$ and

$$
f p-h(a)+f p q\langle d h(a) / q\rangle \equiv\left\{\begin{array}{l}
f p(1+d h(a))-h(a) \equiv p f(\bmod q), \\
-h(a) \equiv 0(\bmod p), \\
-h(a) \equiv-2 a(\bmod f),
\end{array}\right.
$$

we have $r(-a)=f p-h(a)+f p q\langle d h(a) / q\rangle$. The left hand side of $(6)$ is

$$
\begin{aligned}
& \prod_{\substack{0<a<r(a) \\
\chi(a)=-1 \\
q \mid a, p \nmid a}} a=\prod_{\substack{0<-a<r(-a) \\
\chi(a)=1 \\
q \mid a, p \nmid a}}(-a)=\prod_{\substack{0>a>h(a)-f p-f p q\langle d h(a) / q\rangle \\
\chi(a)=1 \\
q \mid a, p \nmid a}}(-a) \\
& =(-1)^{n_{1}} \prod_{y=0}^{q-1}\left(\left(\prod_{\substack{-f p y>a>h(a)-f p-f p y \\
\chi(a)=1, y \equiv d h(a)(\bmod q) \\
q \mid a, p \nmid a}} a\right) . \prod_{\substack{0>a>-f p y \\
\chi(a)=1, y \equiv d h(a)(\bmod q) \\
q \mid a, p \nmid a}} a\right),
\end{aligned}
$$

where $n_{1}$ again means the number of factors of the product on the left hand side of (6). By means of the substitution $b=a+f p(y+1)$ for the former inner product and of the substitution $b=a+f p x, x=1,2, \ldots, y$, for the latter we obtain the following congruence modulo $p$ :

$$
\begin{aligned}
& \prod_{\substack{0<a<r(a) \\
\chi(a)=-1 \\
q \mid a, p \nmid a}} a \equiv(-1)^{n_{1}} \prod_{y=0}^{q-1}\left(\left(\prod_{\substack{h(b)<b<f p \\
\chi(b)=1, y \equiv d h(b)(\bmod q) \\
p \nmid d, d b \equiv y+1(\bmod q)}} b\right) \cdot \prod_{\substack{x=1 \\
\chi(b)=1, y \equiv d h(b)(\bmod q) \\
p \nmid b, d b \equiv x(\bmod q)}} \prod_{\substack{0<b<f p \\
p \nmid y}} b\right) \\
& =(-1)^{n_{1}}\left(\prod_{\substack{h(b)<b<f p \\
\chi(b)=1, p \nmid b \\
d b \equiv d h(b)+1(\bmod q)}} b\right) \cdot \prod_{\substack{0<b<f p \\
\chi(b)=1, p \nmid b \\
0<\langle d b / q\rangle \leq\langle d h(b) / q\rangle}} b(\bmod p) .
\end{aligned}
$$

Let us consider the following partition of the set $I=\left\{a \bmod ^{\times} f p \mid \chi(a)=1\right\}$ :

$$
\begin{aligned}
& I_{1}=\{a \in I|q| a, a<h(a)\}, \\
& I_{2}=\{a \in I|q| a, a>h(a), d h(a) \equiv-1(\bmod q)\}, \\
& I_{3}=\{a \in I|q| a, a>h(a), d h(a) \not \equiv-1(\bmod q)\}, \\
& I_{4}=\{a \in I \mid q \nmid a, a<h(a),\langle d a / q\rangle \leq\langle d h(a) / q\rangle\}, \\
& I_{5}=\{a \in I \mid q \nmid a, a<h(a),\langle d a / q\rangle>\langle d h(a) / q\rangle\}, \\
& I_{6}=\{a \in I \mid q \nmid a, a>h(a), d h(a) \equiv-1(\bmod q)\},
\end{aligned}
$$




$$
\begin{gathered}
I_{7}=\{a \in I \mid q \nmid a, a>h(a), d h(a) \neq \equiv-1(\bmod q), \\
\langle d a / q\rangle \leq\langle(1+d h(a)) / q\rangle\}, \\
I_{8}=\{a \in I \mid q \nmid a, a>h(a), d h(a) \neq \equiv-1 \quad(\bmod q), \\
\langle d a / q\rangle>\langle(1+d h(a)) / q\rangle\} .
\end{gathered}
$$

Tedious evaluation of all possibilities shows that Proposition 6.2 can be stated also in the following form:

$$
(-1)^{n_{1}} v \cdot \prod_{a \in I_{3}} a^{2} \cdot \prod_{a \in I_{1} \cup I_{2} \cup I_{8}} a \cdot \prod_{a \in I_{4}} a^{-1} \equiv 1(\bmod p) .
$$

Let us consider the involution $\varepsilon$ on the set $I$ such that $\varepsilon(a) \equiv a(\bmod f)$ and $\varepsilon(a) \equiv-a(\bmod p)$. Then

$$
n^{2} \equiv \pm \prod_{\substack{a \bmod ^{\times} f p \\ q \mid a, \chi(a)=1}} a^{2} \cdot \prod_{\substack{a \bmod ^{\times} f p \\ \chi(a)=1}} a^{q\langle d a / q\rangle+q\langle d \varepsilon(a) / q\rangle-2}(\bmod p) .
$$

Wilson's theorem implies

$$
\prod_{\substack{a \bmod ^{\times} f p \\ \chi(a)=1}} a^{q\langle d h(a) / q\rangle-1}=\prod_{\substack{t \bmod ^{\times} f \\ \chi(t)=1}}\left(\prod_{\substack{a \bmod ^{\times} f p \\ a \equiv t(\bmod f)}} a\right)^{q\langle\operatorname{mo}(t) / q\rangle-1} \equiv \pm 1(\bmod p) .
$$

Therefore

$$
\text { (8) } n^{2} \equiv \pm \prod_{\substack{a \bmod ^{\times} f p \\ q \mid a, \chi(a)=1}} a^{2} \cdot \prod_{\substack{a \bmod ^{\times} f p \\ \chi(a)=1}} a^{q(\langle d a / q\rangle+\langle d \varepsilon(a) / q\rangle-\langle d h(a) / q\rangle)-1}(\bmod p) .
$$

It is easy to see that

$$
a+\varepsilon(a)= \begin{cases}h(a) & \text { if } a<h(a) \\ h(a)+f p & \text { otherwise }\end{cases}
$$

and so

$$
\langle d(a+\varepsilon(a)) / q\rangle= \begin{cases}\langle d h(a) / q\rangle & \text { if } a<h(a) \\ \langle(1+d h(a)) / q\rangle & \text { otherwise. }\end{cases}
$$

Moreover

$$
\langle d a / q\rangle+\langle d \varepsilon(a) / q\rangle= \begin{cases}\langle d(a+\varepsilon(a)) / q\rangle & \text { if }\langle d a / q\rangle \leq\langle d(a+\varepsilon(a)) / q\rangle \\ \langle d(a+\varepsilon(a)) / q\rangle+1 & \text { otherwise }\end{cases}
$$

and

$$
\langle(1+d h(a)) / q\rangle-\langle d h(a) / q\rangle= \begin{cases}1 / q & \text { if } d h(a) \not \equiv-1(\bmod q) \\ (1-q) / q & \text { otherwise }\end{cases}
$$


If we go again through all eight cases, we find

$$
q(\langle d a / q\rangle+\langle d \varepsilon(a) / q\rangle-\langle d h(a) / q\rangle)-1= \begin{cases}-1 & \text { if } a \in I_{1} \cup I_{4}, \\ 0 & \text { if } a \in I_{3} \cup I_{6} \cup I_{7}, \\ -q & \text { if } a \in I_{2}, \\ q-1 & \text { if } a \in I_{5}, \\ q & \text { if } a \in I_{8},\end{cases}
$$

and (8) gives the following congruence modulo $l$ th powers:

$$
n^{2} \equiv_{l} \pm \prod_{\substack{a \bmod ^{\times} f p \\ q \mid a, \chi(a)=1}} a^{2} \cdot \prod_{a \in I_{1} \cup I_{2} \cup I_{4}} a^{-1} \cdot \prod_{a \in I_{8}} a(\bmod p) .
$$

The lemma follows from (7) and the fact that both -1 and $v$ are $l$ th powers modulo $p$.

Proof of Theorem 6.1. As a first step, we define $a(u, v)$ to be the integer between 0 and $f p q-1$ which is congruent to $u$ modulo $f p$, and congruent to $v$ modulo $q$. Then by definition

$$
f p q R_{1,2}=\sum_{u \in I} \sum_{v=1}^{q-1}\left(a(u, v)-\frac{1}{2} f p q\right) \cdot \lg \sigma_{u} \otimes \lg \tau_{v}
$$

where $I=\left\{u \bmod ^{\times} f p \mid \chi(u)=1\right\}$. For any $u \in I$ and any positive integer $v<q$ let $b(u, v)=(a(u, v)-u) /(f p)$. It is easy to see that $b(u, v)$ is an integer and $0 \leq b(u, v)<q$. Moreover $f p \cdot b(u, v) \equiv v-u(\bmod q)$. Recall that the integer $d$ satisfies $f p d \equiv 1(\bmod q)$, so $b(u, v) \equiv d(v-u)(\bmod q)$, which gives

$$
\begin{aligned}
b(u, v) & =q\langle d(v-u) / q\rangle \\
& =d(v-u)-q[d(v-u) / q]=d(v-u)-q\left([d v / q]+[-d u / q]+\alpha_{u, v}\right),
\end{aligned}
$$

where $\alpha_{u, v}=1$ if $\langle d v / q\rangle+\langle-d u / q\rangle \geq 1$ and $\alpha_{u, v}=0$ otherwise. Therefore

$$
\begin{aligned}
a(u, v) & =u+f p \cdot b(u, v) \\
& =f p(d v-q[d v / q])-f p(d u+q[-d u / q])+u-f p q \alpha_{u, v} .
\end{aligned}
$$

Wilson's theorem shows that $\sum_{v=1}^{q-1} \lg \tau_{v}=\lg \tau_{-1}=0$ and $\sum_{u \in I} \lg \sigma_{u}=$ $\lg \sigma_{-1}=0$. Consequently, any sum of the form $\sum_{u \in I} \sum_{v=1}^{q-1} c(u, v) \lg \sigma_{u} \otimes \lg \tau_{v}$ where the function $c(u, v)$ is independent of $u$ or independent of $v$ will be zero. This shows, given the above expression for $a(u, v)$, that

$$
R_{1,2}=-\sum_{u \in I} \sum_{v=1}^{q-1} \alpha_{u, v} \lg \sigma_{u} \otimes \lg \tau_{v}=\sum_{v=1}^{q-1} \sum_{u \in I}\left(1-\alpha_{u, v}\right) \lg \sigma_{u} \otimes \lg \tau_{v} .
$$

Since $\alpha_{u, v}=0$ if and only if $\langle d v / q\rangle<1-\langle-d u / q\rangle$, which is the case if and only if either $q \mid u$ or $\langle d v / q\rangle<\langle d u / q\rangle$, we obtain 


$$
\begin{aligned}
R_{1,2} & =\sum_{v=1}^{q-1}\left(\sum_{\substack{u \in I \\
q \mid u}} \lg \sigma_{u}+\sum_{\substack{u \in I \\
\langle d u / q\rangle>\langle d v / q\rangle}} \lg \sigma_{u}\right) \otimes \lg \tau_{v} \\
& =\sum_{b=1}^{q-2} \sum_{\substack{u \in I \\
\langle d u / q\rangle>b / q}} \lg \sigma_{u} \otimes \lg \tau_{f p b},
\end{aligned}
$$

where we have used the identity $\sum_{v=1}^{q-1} \lg \tau_{v}=0$ and the substitution $v=f p b$. Since $\gamma(u)=\Gamma_{q}(u / f p) \equiv \Gamma_{q}(d u)(\bmod q)$ and $\Gamma_{q}(a)=(-1)^{a}(a-1) !$ for any $a \in\{1, \ldots, q\}$, the right hand side of the equality in Theorem 6.1 equals

$$
\begin{aligned}
\sum_{u \in I} \lg \sigma_{u} \otimes \lg \tau_{\gamma(u)} & =\sum_{a=1}^{q-1} \sum_{\substack{u \in I \\
d u \equiv a(\bmod q)}} \lg \sigma_{u} \otimes \lg \tau_{(a-1) !} \\
& =\sum_{a=1}^{q-1} \sum_{b=1}^{a-1} \sum_{\substack{u \in I \\
d u \equiv a(\bmod q)}} \lg \sigma_{u} \otimes \lg \tau_{b} \\
& =\sum_{b=1}^{q-2} \sum_{a=b+1}^{q-1} \sum_{\substack{u \in I \\
d u \equiv a(\bmod q)}} \lg \sigma_{u} \otimes \lg \tau_{b} \\
& =\sum_{b=1}^{q-2} \sum_{\substack{u \in I \\
\langle d u / q\rangle>b / q}} \lg \sigma_{u} \otimes \lg \tau_{b} .
\end{aligned}
$$

We have $\lg \tau_{f p b}=\lg \tau_{b}+\lg \tau_{f p}$, so to prove Theorem 6.1 we need to show that

$$
\left(\sum_{b=1}^{q-2} \sum_{\substack{u \in I \\\langle d u / q\rangle>b / q}} \lg \sigma_{u}\right) \otimes \lg \tau_{f p}=0 .
$$

It is easy to see that

$$
\sum_{b=1}^{q-2} \sum_{\substack{u \in I \\\langle d u / q\rangle>b / q}} \lg \sigma_{u}=\sum_{u \in I, q \nmid u}(q\langle d u / q\rangle-1) \lg \sigma_{u} .
$$

We shall show that this sum is zero. In multiplicative notation this amounts to proving that $\prod_{u \in I, q \nmid u} u^{q\langle d u / q\rangle-1}$ is an $l$ th power modulo $p$; but this is exactly the statement of Lemma 6.3.

We return to our numerical example $l=3, f=4, p=13$. (It is not necessary to specify $q$; the dependence on $q$ is hidden in $\gamma$.) Let us identify 
$G_{1}=(\mathbb{Z} / 13 \mathbb{Z})^{\times} / 3$ with $\mathbb{Z} / 3 \mathbb{Z}$ via the following isomorphism $\kappa: \pm 1$ and \pm 5 (the cubes modulo 13 ) map to $\overline{0} ; \pm 2, \pm 10$ map to $\overline{1}$; and $\pm 4, \pm 6$ map to $\overline{2}=\overline{-1}$. Thus $G_{1} \otimes G_{2}$ becomes identified with $G_{2}$, and we can do without the $\lg$ notation. Then $R_{1,2}$, or rather its image in $G_{2}=(\mathbb{Z} / q \mathbb{Z})^{\times} / 3$, turns out to be

$$
\gamma(9)^{-1} \gamma(17)^{-1} \gamma(29) \gamma(33)^{-1} \gamma(37) \gamma(41) \gamma(45)^{-1} \gamma(49) .
$$

Again we do not pretend that this is very enlightening yet. The picture will become clearer in the next section.

7. Establishing the connection with a Gauss sum. In this section we shall assume Assumption A: $s=2$ and $q=p_{2}$ is an $l$ th power modulo $p=p_{1}$. Let $n$ be the order of $q$ modulo $p$. Assumption $\mathrm{A}$ is equivalent to $l \mid r:=(p-1) / n$. Let $\sigma_{q}$ be the Frobenius of $q$ in $L=\mathbb{Q}\left(\zeta_{f p}\right)$.

Let $D_{0} \subseteq F\left(\zeta_{p}\right)$ be the decomposition subfield of $q$, i.e. $\operatorname{Gal}\left(F\left(\zeta_{p}\right) / D_{0}\right)=$ $\left\langle\left.\sigma_{q}\right|_{F\left(\zeta_{p}\right)}\right\rangle$. Assumption A gives $F K_{1} \subseteq D_{0}$. From this point onwards it seems necessary to fix a generator $\widetilde{\sigma}$ of the group $\operatorname{Gal}\left(F\left(\zeta_{p}\right) / F\right)$ and we shall do it in such a way that $\left.\sigma_{q}\right|_{F\left(\zeta_{p}\right)}=\tilde{\sigma}^{r}$. Then the restriction $\sigma_{0}$ of $\widetilde{\sigma}$ to $K_{1}$ is a generator of $G_{1}$, and this choice of generator induces identifications $\lg G_{1} \cong \mathbb{Z} / l \mathbb{Z}$ and $\lg G_{1} \otimes \lg G_{2} \cong \lg G_{2}$, which we will both denote by $\iota^{\prime}$. The lg symbol will sometimes be omitted again (switching back to multiplicative notation when possible), and $G_{2}$ will then be identified with $(\mathbb{Z} / q \mathbb{Z})^{\times}$modulo $l$ th powers, often tacitly.

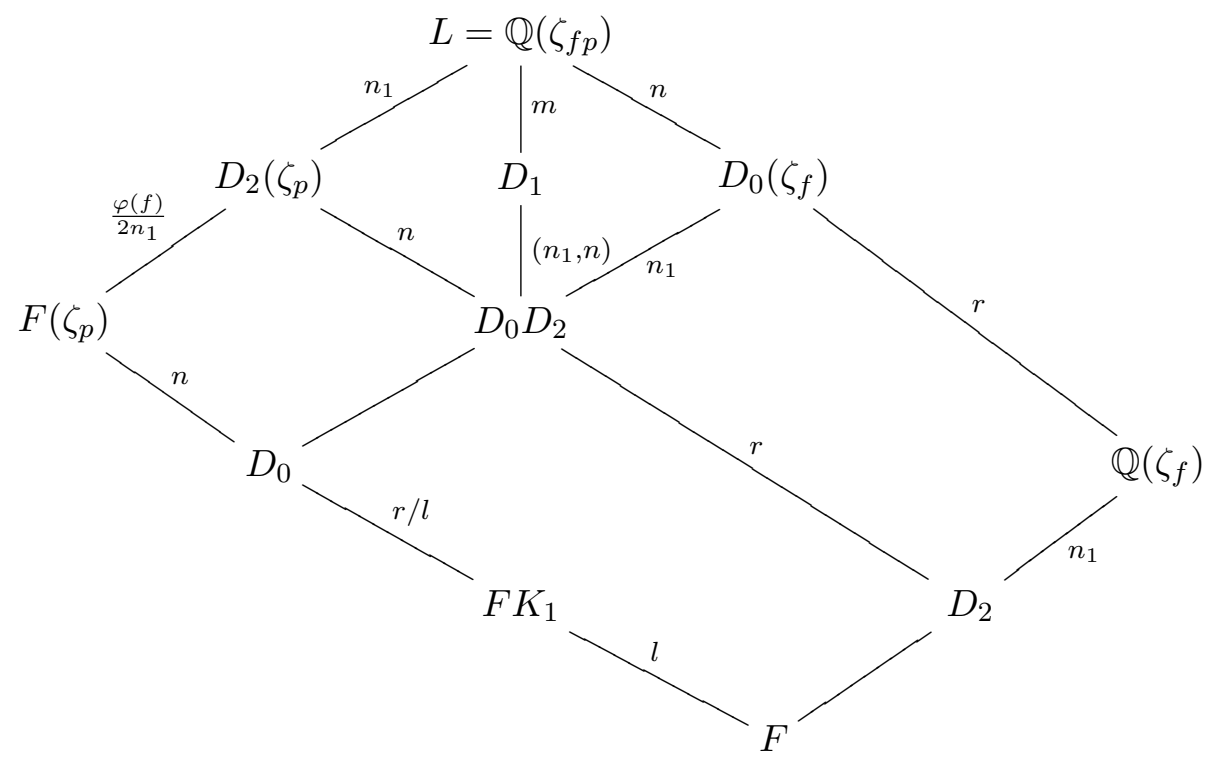

Let $n_{1}$ be the order of $q$ modulo $f$ and let $D_{2} \subseteq \mathbb{Q}\left(\zeta_{f}\right)$ be the decomposition subfield of $q$. Let $m$ be the order of $q$ modulo $f p$, and let $D_{1} \subseteq L$ be the 
decomposition subfield of $q$. So $D_{0} D_{2} \subseteq D_{1}$ and $m$ is the least common multiple of $n$ and $n_{1}$. Then $\left[D_{1}: D_{0}\right]=u:=\varphi(f) n /(2 m)$. The prime $q$ is totally split in $D_{1}$ and we fix a prime $\mathfrak{q}_{1}$ above $q$ in $D_{1}$. Since $\mathfrak{q}_{1}$ stays inert in $L / D_{1}$, we can also view $\mathfrak{q}_{1}$ as a prime in $L$. Let $\psi$ be the additive character on $\mathbb{Z} / q \mathbb{Z}$ sending $\overline{1}$ to $\zeta_{q}$, where the $q$ th primitive root of unity is chosen once and for all. Let $\varrho=\omega^{\left(q^{m}-1\right) /(f p)}$ where $\omega: \mathbb{F}_{q^{m}}^{\times} \rightarrow \mu_{q^{m}-1}$ is the Teichmüller character attached to a prime in $L\left(\zeta_{q^{m}-1}\right)=\mathbb{Q}\left(\zeta_{q^{m}-1}\right)$ above $\mathfrak{q}_{1}$. All we need to know is that the values of $\varrho$ belong to $L$ and that $\varrho\left(x \bmod \mathfrak{q}_{1}\right) \equiv x^{\left(q^{m}-1\right) /(f p)}\left(\bmod \mathfrak{q}_{1}\right)$ for all $x \in \mathcal{O}_{L}$. We shall also need the conjugate characters $\varrho_{v}(x)=\varrho(x)^{v}$ for $v$ running $\bmod ^{\times} f p$. Let $g\left(\varrho_{v}\right)=g\left(\varrho_{v}, \psi \circ \operatorname{Tr}\right) \in L\left(\zeta_{q}\right)$ be the resulting Gauss sums (see e.g. [8, p. 56]). According to [12, p. 97, Lemmas 6.4 and 6.5], $g\left(\varrho_{v}\right) \in D_{1}\left(\zeta_{q}\right)$ and $g\left(\varrho_{v}\right)^{f p} \in D_{1}$.

For any integer $t$ relatively prime to $f$ let $\nu_{t} \in \operatorname{Gal}\left(L / \mathbb{Q}\left(\zeta_{p}\right)\right)$ be determined by $\nu_{t}\left(\zeta_{f}\right)=\zeta_{f}^{t}$. Then

$$
\operatorname{Gal}\left(L / F\left(\zeta_{p}\right)\right)=\left\{\nu_{t} \mid t \bmod ^{\times} f, \chi(t)=1\right\}
$$

and

$$
\operatorname{Gal}\left(L / D_{1}\left(\zeta_{p}\right)\right)=\left\{\nu_{q^{n i}} \mid i=1, \ldots, m / n\right\} .
$$

Let us choose and fix a system of integers $v_{1}, \ldots, v_{u}$ all congruent to 1 modulo $p$ such that

$$
\operatorname{Gal}\left(D_{1}\left(\zeta_{p}\right) / F\left(\zeta_{p}\right)\right)=\left\{\left.\nu_{v_{i}}\right|_{D_{1}\left(\zeta_{p}\right)} \mid i=1, \ldots, u\right\} .
$$

As $D_{1} \cap F\left(\zeta_{p}\right)=D_{0}$, we have $\operatorname{Gal}\left(D_{1} / D_{0}\right) \cong \operatorname{Gal}\left(D_{1}\left(\zeta_{p}\right) / F\left(\zeta_{p}\right)\right)$ via restriction. A key role in the forthcoming Theorem 7.1 is played by

$$
g=\mathrm{N}_{D_{1}\left(\zeta_{q}\right) / D_{0}\left(\zeta_{q}\right)}(g(\varrho))=\prod_{i=1}^{u} g\left(\varrho_{v_{i}}\right) .
$$

The choice of $\psi$ we made entails the choice of a solution $\pi \in \mathbb{Q}_{q}\left(\zeta_{q}\right)$ of the equation $\pi^{q-1}=-q$, as in [8, p. 71]. We may and will identify the local field $\mathbb{Q}_{q}\left(\zeta_{q}\right)$ with $\left(D_{1}\right)_{\mathfrak{q}_{1}}\left(\zeta_{q}\right)$. Let $T: \mathbb{Q}_{q}\left(\zeta_{q}\right)^{\times} \rightarrow \mathcal{O}_{\mathbb{Q}_{q}\left(\zeta_{q}\right)}^{\times}$be the "leading term homomorphism" given by $T(x)=x \pi^{-\operatorname{val}_{\pi}(x)}$. Let $a \equiv_{l} b \in X$ mean that $a / b$ is an $l$ th power in the multiplicative abelian group $X$. Often $X$ will be clear from the context and not mentioned.

We finally define, considering $\widetilde{\sigma}$ as an element of $\operatorname{Gal}\left(D_{0}\left(\zeta_{q}\right) / F\left(\zeta_{q}\right)\right)$ in the obvious way (i.e. $\zeta_{q}^{\widetilde{\sigma}}=\zeta_{q}$ ),

$$
\Delta=\sum_{i=0}^{r-1} i \widetilde{\sigma}^{i} \in \mathbb{Z}\left[\operatorname{Gal}\left(D_{0}\left(\zeta_{q}\right) / F\left(\zeta_{q}\right)\right)\right] .
$$

(Note: This type of element is frequently used in the theory of Euler systems and points to an impending application of Hilbert's Theorem 90.) Our next goal is the following result. 
TheOREM 7.1. Under Assumption A, if the residue map $\mathcal{O}_{\mathbb{Q}_{q}\left(\zeta_{q}\right)} \rightarrow \mathbb{Z} / q \mathbb{Z}$ is written by an overbar, then

$$
\iota^{\prime}\left(R_{1,2}\right) \equiv_{l} \overline{T\left(g^{-\Delta}\right)} \in(\mathbb{Z} / q \mathbb{Z})^{\times} .
$$

Proof. In order to prove this we need the Gross-Koblitz formula (see p. 82 in [8]). Let us point out right away that our $q$ (which is a prime!) and $q^{m}$ correspond to $p$ and $q$ in loc.cit.

The Gross-Koblitz formula states the following equality in $\mathbb{Q}_{q}\left(\zeta_{q}\right)$, where $S$ means the sum of $q$-adic digits:

$$
g\left(\varrho_{v}\right)=\pi^{(q-1) m-S\left(\langle v /(f p)\rangle\left(q^{m}-1\right)\right)} \prod_{i=0}^{m-1} \Gamma_{q}\left(1-\left\langle\frac{q^{i} v}{f p}\right\rangle\right)
$$

and so by means of the functional equation

$$
T\left(g\left(\varrho_{v}\right)\right)= \pm \prod_{i=0}^{m-1} \Gamma_{q}\left(\left\langle\frac{q^{i} v}{f p}\right\rangle\right)^{-1} .
$$

Let $h \equiv 1(\bmod f)$ be such that $\widetilde{\sigma}\left(\zeta_{p}\right)=\zeta_{p}^{h}$ (so $h$ is a primitive root modulo $p$ and $\left.h^{r} \equiv q(\bmod p)\right)$. Let $v(a)$ be the smallest positive residue of $a$ modulo $f p$. We now look at $\iota^{\prime}\left(R_{1,2}\right)$ in $\lg G_{2}$. From Theorem 6.1 we have, replacing $a$ by $v\left(h^{i} t\right)$ and noting that $\iota^{\prime}\left(\sigma_{h^{i} t}\right)=i$,

$$
\iota^{\prime}\left(R_{1,2}\right)=\sum_{\substack{t \bmod ^{\times} f p \\ \chi(t)=1, t \equiv 1(\bmod p)}} \sum_{i=0}^{p-2} i \lg \tau_{\gamma\left(v\left(h^{i} t\right)\right)},
$$

where $\gamma(v)=\Gamma_{q}(v /(f p))$ from $\S 6$. If we identify $G_{2}$ with $(\mathbb{Z} / q \mathbb{Z})^{\times} / l$ and revert to multiplicative notation, this gives

$$
\iota^{\prime}\left(R_{1,2}\right) \equiv_{l} \prod_{\substack{t \bmod ^{\times} p \\ \chi(t)=1, t \equiv 1(\bmod p)}} \prod_{i=0}^{p-2} \overline{\Gamma_{q}\left(\left\langle\frac{h^{i} t}{f p}\right\rangle\right)^{i}} \in(\mathbb{Z} / q \mathbb{Z})^{\times} .
$$

We now calculate the right hand side of the congruence in Theorem 7.1 using (9):

$$
\begin{aligned}
T\left(g^{-\Delta}\right) & =\prod_{i=1}^{u} \prod_{j=0}^{r-1} T\left(g\left(\varrho_{v_{i} h^{j}}\right)\right)^{-j} \\
& = \pm \prod_{i=1}^{u} \prod_{j=0}^{r-1} \prod_{a=0}^{n-1} \prod_{b=0}^{m / n-1} \Gamma_{q}\left(\left\langle\frac{q^{b n+a} v_{i} h^{j}}{f p}\right\rangle\right)^{j}
\end{aligned}
$$




$$
\begin{gathered}
= \pm \prod_{\substack{t \bmod _{f p} \\
\chi(t)=1, t \equiv 1(\bmod p)}} \prod_{j=0}^{r-1} \prod_{a=0}^{n-1} \Gamma_{q}\left(\left\langle\frac{q^{a} h^{j} t}{f p}\right\rangle\right)^{j} \\
= \pm \prod_{\substack{t \bmod _{f} f p \\
\chi(t)=1, t \equiv 1(\bmod p)}} \prod_{j=0}^{r-1} \prod_{a=0}^{n-1} \Gamma_{q}\left(\left\langle\frac{h^{r a+j} t}{f p}\right\rangle\right)^{j} .
\end{gathered}
$$

We have $l \mid r$ and $-1 \equiv_{l} 1$, so if we put $k=r a+j$ and note that then $k \equiv j$ modulo $l$, we obtain

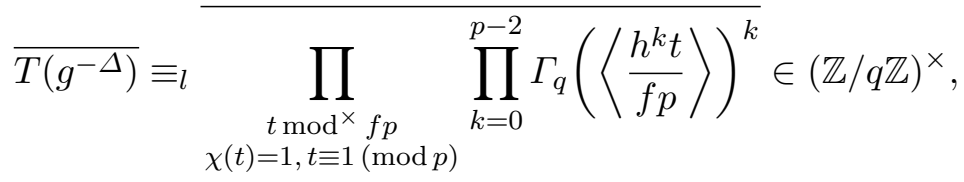

and the theorem follows.

8. The final calculation. In this section we assemble our earlier results and prove the formula given in Corollary 5.5 by an application of Hilbert's Theorem 90. We assume Assumption A and we keep all the notations like $L, D_{0}, D_{1}, g, \Delta$ from the preceding section. As in $\S 3$ we impose that $l \nmid f$, which is slightly stronger than the blanket assumption $l \nmid w_{F}$.

The main technical task is to describe the element $y:=g^{-2 f p w_{F} \Delta} \in D_{0}$ as explicitly as possible. Actually we will work with the slightly modified element $y_{1}=g^{-f p w_{F}(1-\tau) \Delta} \in D_{0}$.

Lemma 8.1. $T\left(y_{1}\right)=T(y)$.

Proof. From the well-known fact $g(\varrho)^{1+\tau}=q^{m}$ we deduce that $g^{1+\tau}=$ $q^{m u}$ and that

$$
y_{1} / y=\left(g^{-1+\tau} / g^{-2}\right)^{f p w_{F} \Delta}=\left(q^{m u}\right)^{f p w_{F} \Delta}=(-q)^{m u f p w_{F} \Delta} .
$$

The lemma follows from $-q=\pi^{q-1}$.

We will show that $y_{1}$ can be written as an $l$ th power times an element $y_{2}$ of $F$, and we will determine the prime factorization of the ideal $\left(y_{2}\right)$, which will turn out to be (modulo the $l$ th power of an ideal of $F$ ) an ideal supported only on prime ideals dividing $p$ and $q$. Now for the details.

We have mentioned that $g(\varrho)^{f p}$ lies in $D_{1}$ and by Stickelberger's theorem the principal ideal $\left(g(\varrho)^{f p}\right)$ has the following prime factorization in $L$ (or in $\left.D_{1}\right)$ :

$$
\left(g(\varrho)^{f p}\right)=\mathfrak{q}_{1}^{f p(\nu-\Theta(L / \mathbb{Q}))},
$$

where $\nu$ is the norm element of $\operatorname{Gal}(L / \mathbb{Q})$ and $f p \Theta(L / \mathbb{Q})=\sum_{a \bmod ^{\times}} a \sigma_{a}^{-1}$, $\sigma_{a}$ being the automorphism of $L$ sending each root of unity to its $a$ th power. (The reason for the minus sign in the exponent and the presence of $\nu$ is that 
in [12], where the exponent is simply $f p \Theta(L / \mathbb{Q})$, one takes negative powers of the Teichmüller character, and we take positive powers. Alternatively, consult the Gross-Koblitz formula in [8].)

Therefore

$$
\left(g(\varrho)^{f p(1-\tau)}\right)=\mathfrak{q}_{1}^{-2 f p(1-\tau) \widetilde{\Theta}_{D_{1}}},
$$

with

$$
\widetilde{\Theta}_{D_{1}}=\sum_{\substack{a \bmod ^{\times} f p \\ \chi(a)=1}}\left(\frac{a}{f p}-\frac{1}{2}\right)\left(\left.\sigma_{a}\right|_{D_{1}}\right)^{-1} \in \mathbb{Q}\left[\operatorname{Gal}\left(D_{1} / F\right)\right] .
$$

Let $\mathfrak{q}_{0}$ be the prime in $D_{0}$ below $\mathfrak{q}_{1}$. As $g^{f p}=\mathrm{N}_{D_{1} / D_{0}}\left(g(\varrho)^{f p}\right)$, the previous decomposition gives

$$
\left(g^{f p(1-\tau)}\right)=\mathfrak{q}_{0}^{-2 f p(1-\tau) \widetilde{\Theta}_{D_{0}}}
$$

with

$$
\widetilde{\Theta}_{D_{0}}=\operatorname{res}_{D_{1} / D_{0}} \widetilde{\Theta}_{D_{1}}=\sum_{\substack{a \bmod ^{\times} f p \\ \chi(a)=1}}\left(\frac{a}{f p}-\frac{1}{2}\right)\left(\left.\sigma_{a}\right|_{D_{0}}\right)^{-1} \in \mathbb{Q}\left[\operatorname{Gal}\left(D_{0} / F\right)\right] .
$$

Then $\widetilde{\Theta}_{D_{0}}$ is a preimage in $\mathbb{Q}\left[\operatorname{Gal}\left(D_{0} / F\right)\right]$ of $\widetilde{\Theta}_{F K_{1}} \in \mathbb{Q}\left[\operatorname{Gal}\left(F K_{1} / F\right)\right]$, which was introduced in $\S 1$. We can easily check that $\widetilde{\Theta}_{D_{0}}$ is divisible by $\widetilde{\sigma}-1$, and write

$$
2 f p \widetilde{\Theta}_{D_{0}} \equiv i_{1}(\widetilde{\sigma}-1)\left(\bmod (\widetilde{\sigma}-1)^{2}\right)
$$

in $\mathbb{Z}\left[\operatorname{Gal}\left(D_{0} / F\right)\right]$. This implies that

$$
2 f p \widetilde{\Theta}_{F K_{1}} \equiv i_{1}\left(\sigma_{0}-1\right) \equiv \sigma_{0}^{i_{1}}-1\left(\bmod \left(\sigma_{0}-1\right)^{2}\right) .
$$

Thus $\iota\left(2 f p \widetilde{\Theta}_{F K_{1}}\right)=\sigma_{0}^{i_{1}}$ (recall $\iota$ is the canonical isomorphism $I_{G_{1}} / I_{G_{1}}^{2}$ $\rightarrow G_{1}$ ), and hence

$$
\iota^{\prime} \iota\left(2 f p \widetilde{\Theta}_{F K_{1}}\right)=i_{1} \in \mathbb{Z} / l \mathbb{Z} .
$$

Thus, up to identifications and multiplying by $2 f p, i_{1}$ is the "leading term" of the minus Stickelberger element attached to $F K_{1}$.

The following simple fact is well-known and easily checked.

Lemma 8.2. $(\widetilde{\sigma}-1) \Delta=r-\mathrm{N}_{\widetilde{\sigma}} \in \mathbb{Z}\left[\operatorname{Gal}\left(D_{0} / F\right)\right]$, where $\mathrm{N}_{\widetilde{\sigma}}=\sum_{i=0}^{r-1} \widetilde{\sigma}^{i}$.

From formula (10) and the fact that the exponent to which $\mathfrak{q}_{0}$ is raised is divisible by $\widetilde{\sigma}-1$, we see that $\mathrm{N}_{\widetilde{\sigma}}\left(g^{f p(1-\tau)}\right)$ is a unit of $F$, that is, a $w_{F}$ th root of unity. From this and Hilbert's Theorem 90 applied to $g^{f p w_{F}(1-\tau)}$ we obtain:

Proposition 8.3. There exists $z_{0}$ in $D_{0}^{\times}$such that $z_{0}^{\widetilde{\sigma}-1}=g^{f p w_{F}(1-\tau)}$. 
Our aim is to compute $\iota^{\prime}\left(R_{1,2}\right)$. Theorem 7.1 and Lemma 8.1 give

$$
\iota^{\prime}\left(R_{1,2}\right)^{2 f p w_{F}} \equiv_{l} \overline{T\left(y_{1}\right)} \in(\mathbb{Z} / q \mathbb{Z})^{\times} .
$$

From Proposition 8.3 and Lemma 8.2 we get

$$
y_{1}=g^{-f p w_{F}(1-\tau) \Delta}=\left(z_{0}^{\widetilde{\sigma}-1}\right)^{-\Delta}=z_{0}^{\mathrm{N}_{\widetilde{\sigma}}-r}
$$

and $l \mid r$ gives $\overline{T\left(y_{1}\right)} \equiv_{l} \overline{T\left(z_{0}^{\mathrm{N} \widetilde{\sigma}}\right)} \in(\mathbb{Z} / q \mathbb{Z})^{\times}$. We have proved

Corollary 8.4. Let $y_{2}=z_{0}^{\mathrm{N}_{\widetilde{\sigma}}}=\mathrm{N}_{D_{0} / F}\left(z_{0}\right) \in F^{\times}$. Then

$$
\iota^{\prime}\left(R_{1,2}\right)^{2 f p w_{F}} \equiv_{l} \overline{T\left(y_{2}\right)} \in(\mathbb{Z} / q \mathbb{Z})^{\times} .
$$

To obtain $\overline{T\left(y_{2}\right)}$ we now have a look at the prime factorization of $\left(y_{2}\right)$. It is easily seen from (10) that the principal ideal $\left(z_{0}\right)$ is the product of the lift of an ideal in $F$ and of an ideal in $D_{0}$ supported only on prime ideals above $p$ and $q$. Therefore the principal ideal $\left(y_{2}\right)=\left(\mathrm{N}_{D_{0} / F}\left(z_{0}\right)\right)$ is equal (up to $l$ th powers of ideals in $F$ ) to an ideal in $F$ supported only on prime ideals above $p$ and $q$.

It is possible to write $2 f p(1-\tau) \widetilde{\Theta}_{D_{0}}=(1-\tau)(\widetilde{\sigma}-1)\left(i_{1}+\beta\right)$ with $\beta \in(\widetilde{\sigma}-1) \mathbb{Z}\left[\operatorname{Gal}\left(D_{0} / F\right)\right]$. If the above- $q$-part of the principal ideal $\left(z_{0}\right)$ is written $\mathfrak{q}_{0}^{\delta}$ with some $\delta \in \mathbb{Z}\left[\operatorname{Gal}\left(D_{0} / \mathbb{Q}\right)\right]$ then we must have $(\widetilde{\sigma}-1) \delta=$ $-2 f p w_{F}(1-\tau) \widetilde{\Theta}_{D_{0}}$, and this implies

$$
\delta \equiv-w_{F}(1-\tau)\left(i_{1}+\beta\right)\left(\bmod \mathrm{N}_{\widetilde{\sigma}} \mathbb{Z}[\langle\tau\rangle]\right)
$$

and so

$$
\delta \mathrm{N}_{\widetilde{\sigma}} \equiv-w_{F}(1-\tau) i_{1} \mathrm{~N}_{\widetilde{\sigma}}\left(\bmod l \mathrm{~N}_{\widetilde{\sigma}} \mathbb{Z}[\langle\tau\rangle]\right) .
$$

Let $\mathfrak{p}_{2}$ be the ideal of $F$ below $\mathfrak{q}_{0}$. (The notation $\mathfrak{p}_{2}$ is consistent with the notation in the statement of the Minus Conjecture in $\S 1$; recall $q$ is just another name for $p_{2}$.) Then the above- $q$-part of $\left(y_{2}\right)$ is (letting $\equiv_{l}$ likewise denote equality of ideals up to $l$ th powers of ideals in $F$ )

$$
\left(y_{2}\right)_{q} \equiv_{l} \mathfrak{p}_{2}^{-w_{F}(1-\tau) i_{1}} .
$$

We shall now concentrate on the above- $p$-part of the ideal $\left(y_{2}\right)$ modulo $l$ th powers. The following result is well-known to experts.

Lemma 8.5. Let $\kappa: \mathbb{Q}_{p}\left(\zeta_{p}\right)^{\times} \rightarrow(\mathbb{Z} / p \mathbb{Z})^{\times}$be given by $\kappa(x)=\overline{x^{\widetilde{\sigma}-1}}$. Then the kernel of $\kappa$ is $\mathcal{O}_{\mathbb{Q}_{p}\left(\zeta_{p}\right)}^{\times} \cdot p^{\mathbb{Z}}$, and the following diagram commutes with $\widetilde{\iota}(\widetilde{\sigma})=\overline{1}$, and the left hand vertical map induced by the valuation:

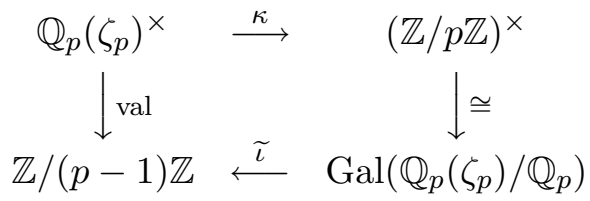


Proof. Recall that $\widetilde{\sigma}$ corresponds to the primitive root $h$ modulo $p$. We have

$$
\kappa\left(\zeta_{p}-1\right)=\overline{\left(\zeta_{p}-1\right)^{\widetilde{\sigma}-1}}=\overline{\left(\zeta_{p}^{h}-1\right) /\left(\zeta_{p}-1\right)}=\overline{\zeta_{p}^{h-1}+\cdots+\zeta_{p}+1}=\bar{h},
$$

and $\operatorname{val}\left(\zeta_{p}-1\right)=1$, so the diagram commutes. The other claims of the lemma follow easily.

To get at the above- $p$-part of the principal ideal $\left(z_{0}\right)$ we need another auxiliary result. Recall that $\varrho=\omega^{\left(q^{m}-1\right) /(f p)}$, where $\omega: \mathbb{F}_{q^{m}}^{\times} \rightarrow \mu_{q^{m}-1}$ is the Teichmüller character attached to a prime $\mathfrak{Q}$ in $\mathbb{Q}\left(\zeta_{q^{m}-1}\right)$ above $\mathfrak{q}_{1}$, and that $n_{1}$ is the order of $q$ modulo $f$. Then $\bar{\omega}=\left.\omega\right|_{\mathbb{F}_{q^{n_{1}}}^{\times}}$is the Teichmüller character attached to the prime below $\mathfrak{Q}$ in $\mathbb{Q}\left(\zeta_{q^{n} 1-1}\right)$. Let $\bar{\varrho}=\bar{\omega}^{\left(q^{n_{1}}-1\right) / f}$ and let $g(\bar{\varrho})$ be the Gauss sum (using the same additive character $\psi$ as in the definition of $g(\varrho)$ ). The relation between these two Gauss sums is described in the following lemma, where $\sigma^{\prime}$ is the automorphism of $\mathbb{Q}\left(\zeta_{f p q}\right)$ determined by $\zeta_{f}^{\sigma^{\prime}}=\zeta_{f}^{p}$ and $\zeta_{p q}^{\sigma^{\prime}}=\zeta_{p q}$. Recall also the notation of $\S 3$ : we have fixed $u_{2} \in F$ such that the prime $\mathfrak{p}_{2}$ below $\mathfrak{Q}$ in $F$ satisfies $\mathfrak{p}_{2}^{h_{F} / h_{F,\left\{p_{2}\right\}}}=\left(u_{2}\right)$.

LEMMA 8.6.

(a) $g(\varrho)^{\sigma^{\prime}} \equiv g(\bar{\varrho})^{m / n_{1}}$ modulo all primes dividing $p$ in $D_{1}\left(\zeta_{q}\right)$.

(b) $z_{0}^{\widetilde{\sigma}-1} \equiv u_{2}^{(1-\tau) 2 n f p h_{F,\left\{p_{2}\right\}}}$ modulo all primes dividing $p$ in $D_{0}$.

Proof. (a) As $\varrho(x)^{p}=\bar{\varrho}\left(\mathrm{N}_{\mathbb{F}_{q} m / \mathbb{F}_{q^{n_{1}}}}(x)\right)$ for any $x \in \mathbb{F}_{q^{m}}^{\times}$, the DavenportHasse relation gives $g\left(\varrho^{p}\right)=g(\bar{\varrho})^{m / n_{1}}$ (e.g., see [12, Ex. 6.4, p. 111]). Let us decompose $\varrho=\varkappa_{p} \varkappa_{f}$ where the characters $\varkappa_{p}, \varkappa_{f}$ satisfy $\varkappa_{p}^{p}=\varkappa_{f}^{f}=1$. Then $\varrho(x)^{p}=\varkappa_{f}(x)^{p}=\varkappa_{f}(x)^{\sigma^{\prime}} \equiv \varrho(x)^{\sigma^{\prime}}\left(\bmod \zeta_{p}-1\right)$ and so $g\left(\varrho^{p}\right) \equiv g(\varrho)^{\sigma^{\prime}}$ $\left(\bmod \zeta_{p}-1\right)$.

(b) We know that $g(\varrho)^{f p} \in D_{1}$ and $g(\bar{\varrho})^{f} \in D_{2}$. It is easy to see that $\left[D_{1}: D_{0} D_{2}\right] \cdot m=n n_{1}$. Thus (a) gives $\mathrm{N}_{D_{1} / D_{0} D_{2}}\left(g(\varrho)^{f p}\right)^{\sigma^{\prime}} \equiv g(\bar{\varrho})^{n f p} \bmod -$ ulo all primes dividing $p$ in $D_{0} D_{2}$. Therefore $g^{f p}=g^{f p \sigma^{\prime}} \equiv \mathrm{N}_{D_{2} / F}\left(g(\bar{\varrho})^{f}\right)^{n p}$ modulo all primes dividing $p$ in $D_{0}$. Stickelberger's theorem gives the factorization

$$
\left(\mathrm{N}_{D_{2} / F}\left(g(\bar{\varrho})^{f}\right)\right)=\mathfrak{p}_{2}^{f\left((1+\tau) \varphi(f) / 2-\Theta_{F}\right)},
$$

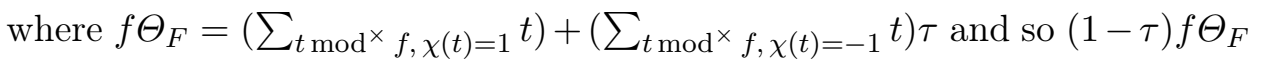
$=(1-\tau) \sum_{t \bmod ^{\times} f} t \chi(t)=-(1-\tau) 2 f h_{F} / w_{F}$. Hence

$$
\left(\mathrm{N}_{D_{2} / F}\left(g(\bar{\varrho})^{f}\right)^{1-\tau}\right)=\mathfrak{p}_{2}^{(1-\tau) 2 f h_{F} / w_{F}}=\left(u_{2}\right)^{(1-\tau) 2 f h_{F,\left\{p_{2}\right\}} / w_{F}},
$$

so the two generators of these principal ideals are equal up to a unit in $F$, i.e. up to a $w_{F}$ th root of unity. We have obtained

$$
\mathrm{N}_{D_{2} / F}\left(g(\bar{\varrho})^{f}\right)^{w_{F}(1-\tau)}=u_{2}^{(1-\tau) 2 f h_{F,\left\{p_{2}\right\}}} .
$$


Therefore, by Proposition 8.3,

$$
z_{0}^{\widetilde{\sigma}-1}=g^{f p w_{F}(1-\tau)} \equiv \mathrm{N}_{D_{2} / F}\left(g(\bar{\varrho})^{f}\right)^{n p w_{F}(1-\tau)}=u_{2}^{(1-\tau) 2 n f p h_{F,\left\{p_{2}\right\}}}
$$

modulo all primes dividing $p$ in $D_{0}$.

Let $\mathfrak{P}_{1}$ and $\mathfrak{P}_{1}^{\prime}$ denote the primes of $D_{0}$ and $F\left(\zeta_{p}\right)$ above $\mathfrak{p}_{1}$, respectively. The extension $F\left(\zeta_{p}\right) / F$ is totally ramified at the primes above $p$ and so

$$
\operatorname{val}_{\mathfrak{p}_{1}}\left(y_{2}\right)=\operatorname{val}_{\mathfrak{p}_{1}}\left(\mathrm{~N}_{D_{0} / F}\left(z_{0}\right)\right)=\operatorname{val}_{\mathfrak{P}_{1}}\left(z_{0}\right)=\frac{1}{n} \operatorname{val}_{\mathfrak{P}_{1}^{\prime}}\left(z_{0}\right) .
$$

Lemma 8.5 gives

$$
\operatorname{val}_{\mathfrak{p}_{1}}\left(y_{2}\right) \equiv \frac{1}{n} \widetilde{\iota}\left(\overline{z_{0}^{\widetilde{\sigma}-1}}\right)\left(\bmod \frac{p-1}{n}\right),
$$

and using Lemma 8.6(b) we obtain

$$
\operatorname{val}_{\mathfrak{p}_{1}}\left(y_{2}\right) \equiv \frac{1}{n} \widetilde{\iota}\left(\left(\overline{u_{2}^{1-\tau}}\right)^{2 n f p h_{F,\left\{p_{2}\right\}}}\right) \equiv 2 f p h_{F,\left\{p_{2}\right\}} \widetilde{\iota}\left(\overline{u_{2}^{1-\tau}}\right)\left(\bmod \frac{p-1}{n}\right) .
$$

The definition of $z_{0}$ in Proposition 8.3 implies that $z_{0}^{(1+\tau)(\widetilde{\sigma}-1)}=1$ and so $z_{0}^{1+\tau} \in F$, which means $z_{0}^{1+\tau} \in \mathbb{Q}$; hence $y_{2}^{1+\tau}=\left(z_{0}^{1+\tau}\right)^{(p-1) / n}$ is an $l$ th power of a rational number. Therefore the above-p-part of $\left(y_{2}\right)$ is

$$
\left(y_{2}\right)_{p} \equiv_{l} \mathfrak{p}_{1}^{2 f p h_{F,\left\{p_{2}\right\}}(1-\tau) \widetilde{\iota}\left(\overline{u_{2}^{1-\tau}}\right)} .
$$

When writing $\widetilde{\iota}(u)$ we tacitly identify $(\mathbb{Z} / p \mathbb{Z})^{\times}$and $\operatorname{Gal}\left(F\left(\zeta_{p}\right) / F\right)$. Since we now work modulo $l$ th powers we actually can write $\iota^{\prime}(u)$ as well (now identifying $(\mathbb{Z} / p \mathbb{Z})^{\times} / l$ and $\left.\operatorname{Gal}\left(F K_{1} / F\right)\right)$. Finally, (12) and (13) give together the following factorization of the principal ideal $\left(y_{2}\right)$ modulo $l$ th powers of ideals in $F$ :

$$
\left(y_{2}\right) \equiv_{l} \mathfrak{p}_{1}^{2 f p h_{F,\left\{p_{2}\right\}}(1-\tau) \iota^{\prime}\left(\overline{u_{2}^{1-\tau}}\right)} \cdot \mathfrak{p}_{2}^{-w_{F}(1-\tau) i_{1}} .
$$

We shall use the notation $t_{1}, t_{2}, t_{3}, t_{1}^{\prime}, t_{2}^{\prime}$, and $\beta_{i j}=\left(u_{i}^{1-\tau},\left(F K_{j}\right)_{\mathfrak{p}_{j}} / F_{\mathfrak{p}_{j}}\right) \in$ $G_{j}$ introduced in $\S 5$. For example, $\left(u_{2}\right)=\mathfrak{p}_{2}^{t_{2}}$, hence $u_{2}^{1-\tau}$ is a unit in $F_{\mathfrak{p}_{1}}$ and the extension $\left(F K_{1}\right)_{\mathfrak{p}_{1}} / F_{\mathfrak{p}_{1}}$ is totally ramified, thus $\beta_{21}^{-1}=\overline{u_{2}^{1-\tau}}$. Now (11) and (MC) for $s=1$ give

$$
-w_{F} i_{1}=\iota^{\prime} \iota\left(-2 f p w_{F} \widetilde{\Theta}_{F K_{1}}\right)=\iota^{\prime} \iota\left(2 f p h_{F,\left\{p_{1}\right\}} \operatorname{Reg}_{F K_{1}, S}^{-}\right)
$$

and using (4) we obtain

$$
\begin{aligned}
-w_{F} i_{1} & =\iota^{\prime} \iota\left(2 f p h_{F, S}\left(t_{2}\left(\beta_{11}-1\right)-t\left(\beta_{21}-1\right)\right)\right) \\
& =2 f p h_{F, S}\left(t_{2} \iota^{\prime}\left(\beta_{11}\right)-t \iota^{\prime}\left(\beta_{21}\right)\right) .
\end{aligned}
$$

Putting things together, we finally arrive at

$$
\left(y_{2}\right) \equiv_{l} \mathfrak{p}_{1}^{-2 f p h_{F, S}(1-\tau) t_{2}^{\prime} \iota^{\prime}\left(\beta_{21}\right)} \cdot \mathfrak{p}_{2}^{2 f p h_{F, S}(1-\tau)\left(t_{2} \iota^{\prime}\left(\beta_{11}\right)-t \iota^{\prime}\left(\beta_{21}\right)\right)}
$$




$$
\begin{aligned}
& \equiv_{l}\left(\mathfrak{p}_{1}^{t_{2}^{\prime}} \mathfrak{p}_{2}^{t}\right)^{-2 f p h_{F, S}(1-\tau) \iota^{\prime}\left(\beta_{21}\right)} \cdot\left(\mathfrak{p}_{2}^{t_{2}}\right)^{2 f p h_{F, S}(1-\tau) \iota^{\prime}\left(\beta_{11}\right)} \\
& =\left(u_{1}^{1-\tau}\right)^{-2 f p h_{F, S} \iota^{\prime}\left(\beta_{21}\right)} \cdot\left(u_{2}^{1-\tau}\right)^{2 f p h_{F, S} \iota^{\prime}\left(\beta_{11}\right)} .
\end{aligned}
$$

Proposition 8.7. If $l \nmid h_{F}$ then (taking lifts of $\iota^{\prime}\left(\beta_{11}\right)$ and $\iota^{\prime}\left(\beta_{21}\right)$ )

$$
y_{2} \equiv_{l}\left(u_{1}^{-(1-\tau) \iota^{\prime}\left(\beta_{21}\right)} \cdot u_{2}^{(1-\tau) \iota^{\prime}\left(\beta_{11}\right)}\right)^{2 f p h_{F, S}} \in F^{\times} .
$$

Proof. We know that there is an ideal $I$ in $F$ such that

$$
\left(y_{2}\right)=I^{l} \cdot\left(u_{1}^{1-\tau}\right)^{-2 f p h_{F, S} \iota^{\prime}\left(\beta_{21}\right)} \cdot\left(u_{2}^{1-\tau}\right)^{2 f p h_{F, S} \iota^{\prime}\left(\beta_{11}\right)} .
$$

Since $l \nmid h_{F}$ and $I^{l}$ is principal, so is $I=(\alpha)\left({ }^{1}\right)$. Then

$$
y_{2} \cdot \alpha^{-l} \cdot\left(u_{1}^{1-\tau}\right)^{2 f p h_{F, S} \iota^{\prime}\left(\beta_{21}\right)} \cdot\left(u_{2}^{1-\tau}\right)^{-2 f p h_{F, S} \iota^{\prime}\left(\beta_{11}\right)}
$$

is a unit in $F$, so a $w_{F}$ th root of unity, which is an $l$ th power in $F$ because $l \nmid w_{F}$. The proposition is proved.

Let us return to the formula (5) (see Corollary 5.5), which we want to prove. When we apply $\iota^{\prime}$ to it and switch to multiplicative notation, it takes the shape

$$
\iota^{\prime}\left(R_{1,2}\right)=\left(\beta_{22}^{\iota^{\prime}\left(\beta_{11}\right)} \cdot \beta_{12}^{-\iota^{\prime}\left(\beta_{21}\right)}\right)^{-h_{F, S} / w_{F}} \in(\mathbb{Z} / q \mathbb{Z})^{\times} / l=G_{2} .
$$

Now $q$ is a norm in $\left(F K_{2}\right)_{\mathfrak{p}_{2}} / F_{\mathfrak{p}_{2}}$ and so

$$
\beta_{22}=\left(u_{2}^{1-\tau},\left(F K_{2}\right)_{\mathfrak{p}_{2}} / F_{\mathfrak{p}_{2}}\right)=\left(u_{2}^{1-\tau} q^{-t_{2}},\left(F K_{2}\right)_{\mathfrak{p}_{2}} / F_{\mathfrak{p}_{2}}\right) \in G_{2} .
$$

Moreover $\left(u_{2}\right)=\mathfrak{p}_{2}^{t_{2}}$, hence $u_{2}^{1-\tau} q^{-t_{2}}$ is a unit in $F_{\mathfrak{p}_{2}}$ and the extension $\left(F K_{2}\right)_{\mathfrak{p}_{2}} / F_{\mathfrak{p}_{2}}$ is totally ramified. Thus $\beta_{22}=\left(\overline{u_{2}^{1-\tau} q^{-t_{2}}}\right)^{-1}$. Recall that the "leading term homomorphism" $T$ was defined as follows: take out the appropriate $\pi$-power from an element of $\mathbb{Q}_{q}\left(\zeta_{q}\right)$ to obtain a unit. As $\underline{\pi^{q-1}=-q \text {, in }}$ $\mathbb{Q}_{q} \cong F_{\mathfrak{p}_{2}}$ we are taking out a power of $-q$ to get a unit, so $\beta_{22}=\overline{ \pm T\left(u_{2}^{1-\tau}\right)^{-1}}$. Similarly we have

$$
\begin{aligned}
\beta_{12} & =\left(u_{1}^{1-\tau},\left(F K_{2}\right)_{\mathfrak{p}_{2}} / F_{\mathfrak{p}_{2}}\right)=\left(u_{1}^{1-\tau} q^{-t},\left(F K_{2}\right)_{\mathfrak{p}_{2}} / F_{\mathfrak{p}_{2}}\right)=\left(\overline{u_{1}^{1-\tau} q^{-t}}\right)^{-1} \\
& = \pm T\left(u_{1}^{1-\tau}\right)^{-1}
\end{aligned}
$$

Therefore (14) is equivalent to

$$
\left.\iota^{\prime}\left(R_{1,2}\right)=\overline{\left(T\left(u_{2}^{(1-\tau) \iota^{\prime}\left(\beta_{11}\right)} \cdot u_{1}^{-(1-\tau) \iota^{\prime}\left(\beta_{21}\right)}\right)\right.}\right)^{h_{F, S} / w_{F}} \in(\mathbb{Z} / q \mathbb{Z})^{\times} / l=G_{2} .
$$

Since $l \nmid 2 f p w_{F}$, this equality follows from Proposition 8.7 and Corollary 8.4. We have proved

TheOrem 8.8. Suppose $s=2, K^{+}$is a genus field (that is, $K^{+}=$ $\left.K_{1} K_{2}\right), p_{2}$ is an lth power modulo $p_{1}$ and $l \nmid f h_{F}$. Then the Minus Conjecture (MC) is true for $K$.

$\left({ }^{1}\right)$ This is the only point in $\S 8$ where we are using $l \nmid h_{F}$. 
REMARK. It follows immediately that Theorem 8.8 remains true if $K^{+}$ is any subfield of $K_{1} K_{2}$ with conductor $p_{1} p_{2}$, since the constructions of both the Stickelberger element and the minus reciprocity matrix are compatible, in an obvious sense, with lowering of the top field, as long as the set of ramified primes does not get any smaller. (This compatibility with lowering of the base field is likewise true for Conjecture (B), but certainly less obvious, since one has to deal with the parameter $\varphi$.)

In conclusion, from Theorem 8.8, the previous remark, and Corollary 2.6 we obtain:

TheOREM 8.9. Suppose $s=2, K^{+}$is cyclic (that is, a subfield of degree $l$ of $K_{1} K_{2}$ different from $K_{1}$ and $\left.K_{2}\right)$, and $l \nmid f h_{F}$. Then (MC) holds for $K$.

\section{References}

[1] D. Burns, Congruences between derivatives of abelian L-functions at $s=0$, Invent. Math. 169 (2007), 451-499.

[2] S. Chaiken and D. J. Kleitman, Matrix tree theorems, J. Combin. Theory Ser. A 24 (1978), 377-381.

[3] H. Darmon, Thaine's method for circular units and a conjecture of Gross, Canad. J. Math. 47 (1995), 302-317.

[4] C. Greither and R. Kučera, The Minus Conjecture revisited, preprint.

[5] B. Gross, On the values of abelian L-functions at $s=0$, J. Fac. Sci. Univ. Tokyo Sect. 1A Math. 35 (1988), 177-197.

[6] A. Hayward, A class number formula for higher derivatives of abelian L-functions, Compos. Math. 140 (2004), 99-129.

[7] -, Congruences satisfied by Stark units, Ph.D. thesis, King's College London, 2004.

[8] N. Koblitz, p-adic Analysis: a Short Course on Recent Work, London Math. Soc. Lecture Note Ser. 46, Cambridge Univ. Press, 1980.

[9] R. Kučera, On the Stickelberger ideal and circular units of a compositum of quadratic fields, J. Number Theory 56 (1996), 139-166.

[10] W. Sinnott, On the Stickelberger ideal and the circular units of an abelian field, Invent. Math. 62 (1980), 181-234.

[11] J. Tate, Les conjectures de Stark sur les fonctions $L$ d'Artin en $s=0$, Notes d'un cours à Orsay rédigées par D. Bernardi et N. Schappacher, Birkhäuser, 1984.

[12] L. Washington, Introduction to Cyclotomic Fields, Grad. Texts in Math. 83, Springer, New York, 1982.

Institut für theoretische Informatik und Mathematik

Fakultät für Informatik

Universität der Bundeswehr München

85577 Neubiberg, Germany

E-mail: cornelius.greither@unibw.de
Př́rodovědecká fakulta

Masarykova univerzita Janáčkovo nám. 2a 60200 Brno, Czech Republic E-mail: kucera@math.muni.cz

Received on 29.12.2006

and in revised form on 24.5.2007 